\title{
Analysis and Design of Numerical Schemes for Gas Dynamics 2: Artificial Diffusion and Discrete Shock Structure
}

\author{
Antony Jameson
}

The Research Institute of Advanced Computer Science is operated by Universities Space Research Association, The American City Building, Suite 212, Columbia, MD 21044, (410) 730-2656

Work reported herein was sponsored by NASA under contract NAS 2-13721 between NASA and the Universities Space Research Association (USRA). 



\title{
Analysis and Design of Numerical Schemes for Gas Dynamics 2 Artificial Diffusion and Discrete Shock Structure
}

\author{
Antony Jameson \\ Department of Mechanical and Aerospace Engineering \\ Princeton University \\ Princeton, New Jersey 08544 USA
}

\begin{abstract}
The effect of artificial diffusion on discrete shock structures is examined for a family of schemes which includes scalar diffusion, convective upwind and split pressure (CUSP) schemes, and upwind schemes with characteristic splitting. The analysis leads to conditions on the diffusive flux such that stationary discrete shocks can contain a single interior point. The simplest formulation which meets these conditions is a CUSP scheme in which the coefficients of the pressure differences is fully determined by the coefficient of convective diffusion. It is also shown how both the characteristic and CUSP schemes can be modified to preserve constant stagnation enthalpy in steady flow, leading to four variants, the $\mathrm{E}$ and $\mathrm{H}$-characteristic schemes, and the $\mathrm{E}$ and H-CUSP schemes. Numerical results are presented which confirm the properties of these schemes.
\end{abstract}

\section{Introduction}

The development of computational methods for the solution of gas dynamic equations has presented a continuing challenge. The goal of combining

1. high accuracy

2. high resolution of shock waves and contact discontinuities without oscillation

3. minimum computational complexity

in a single scheme has proved elusive. Two main issues in the design of non-oscillatory high resolution schemes were identified in the previous paper of this series [5]; first the design of scalar discrete schemes which guarantee the preservation of positivity and monotonicity in the solution, and second the construction of numerical fluxes for systems of equations to allow the proper resolution of complex wave interactions which may lead to the formation of both shock waves and contact discontinuities. The earlier paper develops systematic procedures for the design of scalar discretization schemes which satisfy positivity constraints. The present paper focuses on the design of numerical fluxes for the gas dynamic equations.

Results presented in the previous paper confirm that stationary shocks can be resolved with a single interior point by combining either a symmetric limited positive (SLIP) scheme or an upstream limited positive (USLIP) scheme with a characteristic decomposition of the diffusive flux. The present paper presents an analysis of the conditions under which a discrete stationary shock can contain a single interior point. It emerges that a characteristic decomposition is not necessary to meet these conditions. Perfect single point discrete shocks completely free of oscillations can be produced by simpler flux splittings belonging to the class of convective 
upwind and split pressure (CUSP) schemes, in which scalar diffusion is augmented by pressure differences. It is actually possible to obtain high resolution with almost no oscillation by introducing the right amount of of scalar diffusion, though this seems to result in a scheme which is less robust than the CUSP scheme.

Section 2 reviews the shock jump conditions for one-dimensional flow, and their relationship to Roe's linearization [9]. Section 3 reviews alternative splittings for a family of schemes. In all of them the diffusive flux is defined by a matrix which can be expressed as a polynomial function of the Jacobian matrix. Section 4 examines semi-discrete schemes for the one-dimensional gas dynamic equations, and analyzes the conditions under which the numerical fluxes can be in perfect equilibrium when the discrete shock structure contains one interior point. These constraints can be satisfied by any numerical flux such that the equilibrium across the interface at the exit of the shock corresponds to the Hugoniot equation for a moving shock, while equilibrium across the interface at the entrance to the shock is maintained by full upwinding. The Roe linearization can be used to construct a variety of fluxes with these properties, with or without characteristic decomposition. In steady-state calculations the total enthalpy should be constant. Unfortunately numerical fluxes derived from the standard characteristic decomposition are not compatible with this property. Section 5 shows how the splittings can be modified so that this property is recovered while the discrete shock structure still has a single interior point. Section 6 discusses the implementation of limiters for these schemes. Numerical results which confirm the properties of the schemes are presented in section 7 .

\section{Shock Jump Conditions and Roe Linearization}

The general one dimensional conservation law for a system of equations can be expressed as

$$
\frac{\partial w}{\partial t}+\frac{\partial}{\partial x} f(w)=0
$$

For the equations of gas dynamics the state and the flux vectors are

$$
w=\left(\begin{array}{c}
\rho \\
\rho u \\
\rho E
\end{array}\right), \quad f=\left(\begin{array}{c}
\rho u \\
\rho u^{2}+p \\
\rho u H
\end{array}\right)
$$

where $\rho$ is the density, $u$ is the velocity, $E$ is the total energy, $p$ is the pressure, and $H$ is the stagnation enthalpy. If $\gamma$ is the ratio of specific heats and $c$ is the speed of sound then

$$
\begin{gathered}
p=(\gamma-1) \rho\left(E-\frac{u^{2}}{2}\right) \\
c^{2}=\frac{\gamma p}{\rho} \\
H=E+\frac{p}{\rho}=\frac{c^{2}}{\gamma-1}+\frac{u^{2}}{2} .
\end{gathered}
$$

In a steady flow $H$ is constant. This remains true for the discrete scheme only if the numerical diffusion is constructed so that it is compatible with this condition.

When the flow is smooth it can be represented by the quasi-linear form

$$
\frac{\partial w}{\partial t}+A(w) \frac{\partial w}{\partial x}=0
$$

where $A(w)=\frac{\partial f}{\partial w}$, and the eigenvalues $u, u+c$ and $u-c$ of the Jacobian matrix A are the wave speeds for the three characteristics. In smooth flow the entropy $S$ is constant along streamlines, and in isentropic flow the Riemann invariants $R^{ \pm}=u \pm \frac{2 c}{\gamma-1}$ are constant along the characteristics $\frac{d x}{d t}=u \pm c$. These conditions may be expressed by the three equations,

$$
\frac{\partial R^{+}}{\partial t}+(u+c) \frac{\partial R^{+}}{\partial x}=0
$$




$$
\begin{aligned}
\frac{\partial R^{-}}{\partial t}+(u-c) \frac{\partial R^{-}}{\partial x} & =0 \\
\frac{\partial S}{\partial t}+u \frac{\partial S}{\partial x} & =0 .
\end{aligned}
$$

Taking the dependent variables as

$$
w=\left(\begin{array}{c}
\frac{2 c}{\gamma-1} \\
u \\
S
\end{array}\right),
$$

the equations can be expressed in the symmetric form

$$
\frac{\partial \tilde{w}}{\partial t}+\tilde{A}(\tilde{w}) \frac{\partial \tilde{w}}{\partial x}=0
$$

where

$$
\tilde{A}(\tilde{w})=\left(\begin{array}{ccc}
u & c & 0 \\
c & u & 0 \\
0 & 0 & u
\end{array}\right) .
$$

Depending on the initial data, there may not be a smooth solution of the conservation law (1). Nonlinear wave interactions along converging characteristics may lead to the formation and propagation of shock waves, while contact discontinuities may also appear. Denote the left and right states across a shock by subscripts $L$ and $R$, and let $[f]$ and $[w]$ be the jumps $f_{R}-f_{L}$ and $w_{R}-w_{L}$. The shock jump condition is then

$$
[f]=\mathcal{S}[w],
$$

where $\mathcal{S}$ is the shock speed.

In order to simplify the analysis of the equations when there are finite jumps in $w$ and $f$, Roe introduced the linearization

$$
f_{R}-f_{L}=A_{R L}\left(w_{R}, w_{L}\right)\left(w_{R}-w_{L}\right) .
$$

where $A_{R L}\left(w_{R}, w_{L}\right)$ is a Jacobian matrix calculated from the left and right states in such a way that this relation is exact [9]. He showed that one way to do this is to introduce weighted averages

$$
u=\frac{\sqrt{\rho_{R}} u_{R}+\sqrt{\rho_{L}} u_{L}}{\sqrt{\rho_{R}}+\sqrt{\rho_{L}}}, \quad H=\frac{\sqrt{\rho_{R}} H_{R}+\sqrt{\rho_{L}} H_{L}}{\sqrt{\rho_{R}}+\sqrt{\rho_{L}}}
$$

into all the formulas in the standard expression for the Jacobian matrix $A(w)$. In the case of a shock wave it now follows that

$$
A_{R L}\left(w_{R}-w_{L}\right)=\mathcal{S}\left(w_{R}-w_{L}\right) .
$$

Thus the shock speed $\mathcal{S}$ is an eigenvalue of $A_{R L}$, and the jump $w_{R}-w_{L}$ is an eigenvector. In the case of a stationary shock $S=0$. If we consider flow with $u>0$ only the eigenvalue $u-c$ can be zero. It follows that when $u$ and $c$ are calculated with Roe averages, $u=c$ for a stationary shock.

\section{Alternative Splittings}

Suppose that the conservation law (1) is approximated over the interval $(0, L)$ on a mesh with an interval $\Delta x$ by the semi-discrete scheme

$$
\Delta x \frac{d w_{j}}{d t}+h_{j+\frac{1}{2}}-h_{j-\frac{1}{2}}=0
$$

where $w_{j}$ denotes the value of the discrete solution in cell $j$, and $h_{j+\frac{1}{2}}$ is the numerical flux between cells $j$ and $j+1$. Let $f_{j}$ denote the flux vector $f\left(w_{j}\right)$ evaluated for the state $w_{j}$. Suppose also that the numerical flux is

$$
h_{j+\frac{1}{2}}=\frac{1}{2}\left(f_{j+1}+f_{j}\right)-d_{j+\frac{1}{2}}
$$


where $d_{j+\frac{1}{2}}$ is a diffusive flux which is introduced to enable the scheme to resolve discontinuities without producing oscillations in the discrete solution. The diffusive flux is assumed to have the form

$$
d_{j+\frac{1}{2}}=\frac{1}{2} \alpha_{j+\frac{1}{2}} B_{j+\frac{1}{2}}\left(w_{j+1}-w_{j}\right)
$$

where the matrix $B_{j+\frac{1}{2}}$ determines the properties of the scheme, and the scaling factor $\alpha_{j+\frac{1}{2}}$ is included for convenience. Introducing a Roe linearization, let $A_{j+\frac{1}{2}}\left(w_{j+1}, w_{j}\right)$ be an estimate of the Jacobian matrix $\frac{\partial f}{\partial w}$ with the property that

$A_{j+\frac{1}{2}}$ can be decomposed as

$$
A_{j+\frac{1}{2}}\left(w_{j+1}-w_{j}\right)=f_{j+1}-f_{j}
$$

$$
A_{j+\frac{1}{2}}=T \Lambda T^{-1}
$$

where the columns of $T$ are the eigenvectors of $A_{j+\frac{1}{2}}$, and $\Lambda$ is a diagonal matrix containing its eigenvalues. Then the upwind scheme is produced by setting

$$
B_{j+\frac{1}{2}}=\left|A_{j+\frac{1}{2}}\right|=T|\Lambda| T^{-1},
$$

where the notation $\left|A_{j+\frac{1}{2}}\right|$ is used to represent the absolute value of $A_{j+\frac{1}{2}}$ which is defined to be the matrix obtained by replacing the eigenvalues by their absolute values. Scalar diffusion is produced by setting

$$
B_{j+\frac{1}{2}}=1 \text {. }
$$

An intermediate class of schemes can be formulated by defining the first order diffusive flux as a combination of differences of the state and flux vectors

$$
d_{j+\frac{1}{2}}=\alpha_{j+\frac{1}{2}}\left(w_{j+1}-w_{j}\right)+\beta_{j+\frac{1}{2}}\left(f_{j+1}-f_{j}\right) .
$$

Schemes of this class are fully upwind in supersonic flow if one takes $\alpha_{j+\frac{1}{2}}=0$ and $\beta_{j+\frac{1}{2}}=\operatorname{sign}(M)$ when the absolute value of the Mach number $M$ exceeds 1 . The flux vector $f$ can be decomposed as

$$
f=u w+f_{p}
$$

where

$$
f_{p}=\left(\begin{array}{c}
0 \\
p \\
u p
\end{array}\right)
$$

Then

$$
f_{j+1}-f_{j}=\bar{u}\left(w_{j+1}-w_{j}\right)+\bar{w}\left(u_{j+1}-u_{j}\right)+f_{p_{j+1}}-f_{p_{j}}
$$

where $\bar{u}$ and $\bar{w}$ are the arithmetic averages

$$
\bar{u}=\frac{1}{2}\left(u_{j+1}+u_{j}\right), \quad \bar{w}=\frac{1}{2}\left(w_{j+1}+w_{j}\right) .
$$

All these schemes can be obtained by representing $B_{j+\frac{1}{2}}$ as a polynomial in the matrix $A_{j+\frac{1}{2}}$ defined by equation (5). According to the Cayley-Hamilton theorem, a matrix satisfies its own characteristic equation. Therefore the third and higher powers of $A$ can be eliminated, and there is no loss of generality in limiting $B_{j+\frac{1}{2}}$ to a polynomial of degree 2 ,

$$
B_{j+\frac{1}{2}}=\alpha_{0} I+\alpha_{1} A_{j+\frac{1}{2}}+\alpha_{2} A_{j+\frac{1}{2}}^{2} .
$$

The characteristic upwind scheme for which $B_{j+\frac{1}{2}}=\left|A_{j+\frac{1}{2}}\right|$ is obtained by substituting $A_{j+\frac{1}{2}}=T \Lambda T^{-1}$, $A_{j+\frac{1}{2}}^{2}=T \Lambda^{2} T^{-1}$. Then $\alpha_{0}, \alpha_{1}$, and $\alpha_{2}$ are determined from the three equations

$$
\alpha_{0}+\alpha_{1} \lambda_{k}+\alpha_{2} \lambda_{k}^{2}=\left|\lambda_{k}\right|, \quad k=1,2,3 .
$$

The same representation remains valid for three-dimensional flow because $A_{j+\frac{1}{2}}$ still has only three distinct eigenvalues $u, u+c, u-c$.

Since $w_{j+1}-w_{j}$ approximates $\Delta x \frac{\partial w}{\partial x}$, the diffusive flux introduces an error proportional to the mesh width, and both these schemes will be first order accurate unless compensating anti-diffusive terms are introduced. 


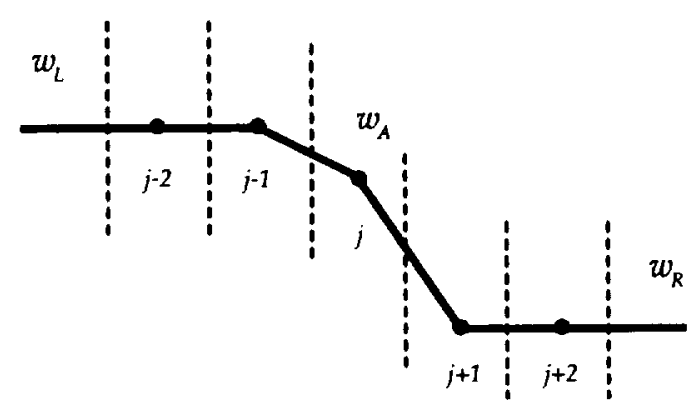

Figure 1: Shock structure for single interior point.

\section{Conditions for a Stationary Shock}

The model of a discrete shock which will be examined is illustrated in figure (1). Suppose that $w_{L}$ and $w_{R}$ are left and right states which satisfy the jump conditions for a stationary shock, and that the corresponding fluxes are $f_{L}=f\left(w_{L}\right)$ and $f_{R}=f\left(w_{R}\right)$. Since the shock is stationary $f_{L}=f_{R}$. The ideal discrete shock has constant states $w_{L}$ to the left and $w_{R}$ to the right, and a single point with an intermediate value $w_{A}$. The intermediate value is needed to allow the discrete solution to correspond to a true solution in which the shock wave does not coincide with an interface between two mesh cells. According to equation (1)

$$
\int_{0}^{L} w(T) d x=\int_{0}^{L} w(0) d x+\int_{0}^{T}\left(f_{R B}-f_{L B}\right) d t
$$

where $f_{L B}$ and $f_{R B}$ are the fluxes at the left and right boundaries. Assuming that the boundary conditions are compatible with a steady solution containing a stationary shock, the location $x_{s}$ of the shock is fixed by this equation, since

$$
\int_{0}^{L} w(T) d x=x_{s} w_{L}+\left(L-x_{s}\right) w_{R}
$$

Similarly in the semi-discrete system

$$
\sum_{j} w_{j}(T)=\sum_{j} w_{j}(0)+\int_{0}^{T}\left(f_{R B}-f_{L B}\right) d t .
$$

Thus $\sum_{j} w_{j}(T)$ has a value which is determined by the initial and boundary conditions, and in general it is not possible for this value to be attained by a discrete solution without an intermediate point, because then the sum would be quantized, increasing by $w_{R}-w_{L}$ whenever the shock location is shifted one cell to the right.

Three diffusion models of varying complexity are examined in the following paragraphs to determine their ability to support the ideal shock structure containing a single interior point. These correspond to one, two or three terms in equation (11).

\subsection{Case 1 Scalar Diffusion}

The first model is simple scalar diffusion with

$$
d_{j+\frac{1}{2}}=\frac{1}{2} \alpha_{j+\frac{1}{2}}\left(w_{j+1}-w_{j}\right) .
$$

Consider the equilibrium in the cell immediately to the right of the shock. Using subscripts $A R$ and $R R$ to denote the values at the cell boundaries, the outgoing flux is

$$
h_{R R}=\frac{1}{2}\left(f_{R}+f_{R}\right)-\frac{1}{2} \alpha_{R R}\left(w_{R}-w_{R}\right)=f_{R}
$$


while the incoming flux is

$$
h_{A R}=\frac{1}{2}\left(f_{R}+f_{A}\right)-\frac{1}{2} \alpha_{A R}\left(w_{R}-w_{A}\right) .
$$

For equilibrium these must be equal. It follows that

$$
f_{R}-f_{A}+\alpha_{A R}\left(w_{R}-w_{A}\right)=0
$$

This is the Hugoniot condition for a shock moving to the left with a speed $\alpha_{A R}$. Introduce a Roe linearization with a mean Jacobian matrix $A_{A R}\left(w_{A}, w_{R}\right)$ such that

$$
f_{R}-f_{A}=A_{A R}\left(w_{R}-w_{A}\right)
$$

Then $w_{R}-w_{A}$ is an eigenvector of $A_{A R}$ corresponding to the eigenvalue $-\alpha_{A R}$. The eigenvalues of $A_{A R}$ are $u, u+c$ and $u-c$. If we consider flow to the right with $u>0$, and $u<c$, a solution with positive numerical diffusion is obtained by taking $\alpha_{A R}=|u-c|$. Then the intermediate value $w_{A}$ must lie on a Hugoniot curve defined by the right state $w_{R}$.

When the corresponding equilibrium is considered for a cell immediately to the left of a shock wave in a flow moving to the left, it is found that the diffusion coefficient should be $|u+c|$. Both cases can be satisfied by taking $\alpha=\min (|u+c|,|u-c|)$. In the neighborhood of a stagnation point the accuracy can be improved by taking $\alpha$ proportional to $u$ to prevent the numerical diffusion becoming undesirably large. This suggests the strategy of using a diffusion coefficient proportional to the smallest eigenvalue, or

$$
\alpha_{j+\frac{1}{2}}=\min _{k}\left|\lambda_{k}\right|
$$

where $\lambda_{k}$ are the eigenvalues $u, u+c$, and $u-c$ of $A_{j+\frac{1}{2}}$. To prevent the scheme from admitting stationary expansion shocks which would violate the entropy condition, the diffusion coefficient may be redefined as

$$
\alpha_{j+\frac{1}{2}}=\min _{k} \hat{\lambda}_{k}
$$

where

$$
\hat{\lambda}_{k}= \begin{cases}\left|\lambda_{k}\right| & \text { if }\left|\lambda_{k}\right| \geq \epsilon \\ \frac{1}{2}\left(\epsilon+\frac{\left|\lambda_{k}\right|^{2}}{\epsilon}\right) & \text { if }\left|\lambda_{k}\right|<\epsilon,\end{cases}
$$

and $\epsilon$ is a positive threshold proportional to $c$. Recent work of Aiso [1] has established that in the scalar case this modification of the viscosity is sufficient to guarantee that the discrete solution will satisfy the entropy condition. The usual strategy in schemes using scalar diffusion has been to make the diffusion coefficient proportional to the maximum eigenvalue of the Jacobian matrix $\frac{\partial f}{\partial w}$, in order to make sure that the numerical viscosity for each characteristic variable is large enough to satisfy the positivity condition. Numerical tests with the alternative strategy of using the smallest eigenvalue confirm that very sharp discrete shocks are obtained, and that the scheme is robust with a viscosity threshold of the type defined by equation (14).

To determine whether scalar diffusion can exactly support an ideal discrete shock it is also necessary to examine the equilibrium in the cell immediately before the shock. In this case the numerical fluxes are

$$
h_{L L}=f_{L}
$$

and

$$
h_{L A}=\frac{1}{2}\left(f_{A}+f_{L}\right)-\frac{1}{2} \alpha_{L A}\left(w_{A}-w_{L}\right) .
$$

For equilibrium it is necessary that

$$
f_{A}-f_{L}-\alpha_{L A}\left(w_{A}-w_{L}\right)=0
$$

which is the Hugoniot condition for a shock moving to the right with a speed $\alpha_{L A}$. Introducing the Roe linearization, $w_{A}-w_{L}$ must now be an eigenvector of $A_{L A}$. The transition from $L$ to $A$, however, is less than 
the full jump for a stationary shock for which it is known that Roe averaging results in $u=c$. Thus it may be expected that $u>c$, and the choice $\alpha_{L A}=u-c=|u-c|$ could still allow the equilibrium condition to be satisfied. Then $w_{A}$ lies on a Hugoniot curve defined by the left state $w_{L}$.

The question now arises whether an intermediate state $w_{A}$ can be found that simultaneously lies on Hugoniot curves defined by the left and right states $w_{L}$ and $w_{R}$, where these two states themselves satisfy the Hugoniot condition for a steady shock. It turns out that this is not possible. Let $v=\frac{1}{\rho}$ be the specific volume. Then all possible shocks connecting $w_{L}$ and $w_{R}$ must satisfy the Hugoniot relation

$$
p_{R} v_{R}-p_{L} v_{L}=\frac{\gamma-1}{2}\left(p_{R}+p_{L}\right)\left(v_{L}-v_{R}\right) \text {. }
$$

This establishes a locus on a $p-v$ diagram of a family of shocks as the shock speed is varied. The single point shock structure requires $w_{A}$ to lie on the Hugoniot curves defined by $w_{L}$ and $w_{R}$. The curve defined from $w_{L}$ is

$$
p_{A} v_{A}-p_{L} v_{L}=\frac{\gamma-1}{2}\left(p_{A}+p_{L}\right)\left(v_{L}-v_{A}\right)
$$

while the curve from $w_{R}$ is

$$
p_{R} v_{R}-p_{A} v_{A}=\frac{\gamma-1}{2}\left(p_{R}+p_{A}\right)\left(v_{A}-v_{R}\right) \text {. }
$$

These intersect only when $w_{A}=w_{R}$ or $w_{L}$. To prove this note that (15) can be written as

$$
p_{R} v_{R}-p_{L} v_{L}=\alpha\left(p_{R} v_{L}-p_{L} v_{R}\right)
$$

where $\alpha=\frac{\gamma-1}{\gamma+1}$. Similarly (16) and (17) yield

$$
p_{A}=p_{L} \frac{v_{L}-\alpha v_{A}}{v_{A}-\alpha v_{L}}=p_{R} \frac{v_{R}-\alpha v_{A}}{v_{A}-\alpha v_{R}} .
$$

Thus $v_{A}$ satisfies a quadratic equation which may be written as

$$
\left(p_{R} v_{R}-p_{L} v_{L}\right) v_{A}-\alpha\left(p_{R}-p_{L}\right)\left(v_{A}^{2}+v_{L} v_{R}\right)+\alpha^{2} v_{A}\left(p_{R} v_{L}-p_{L} v_{R}\right)=0
$$

Substituting from equation (18)

$$
v_{A}\left(p_{R} v_{L}-p_{L} v_{R}\right)-\left(p_{R}-p_{L}\right)\left(v_{A}^{2}+v_{L} v_{R}\right)+v_{A}\left(p_{R} v_{R}-p_{L} v_{L}\right)=0
$$

or

$$
\left(p_{R}-p_{L}\right)\left(v_{A}-v_{R}\right)\left(v_{A}-v_{L}\right)=0 .
$$

If $p_{L} \neq p_{R}$ this has only the solutions $v_{A}=v_{L}$ or $v_{A}=v_{R}$. Therefore it is concluded that scalar diffusion cannot support a perfect discrete shock with a single interior point. Calculations of one-dimensional flows reveal an oscillation of very small amplitude upstream of the shock. In multidimensional flows, however, these oscillations are essentially imperceptible.

\subsection{Case 2 Characteristic Upwind Scheme}

The second case to be examined is the upwind scheme which results from characteristic decomposition, with $B_{j+\frac{1}{2}}=\left|A_{j+\frac{1}{2}}\right|$. This case has been studied by Roe [10], and it is known that the upwind scheme admits ideal shocks. Assuming flow to the right with $u>0$, the fluxes in the cell to the right of the shock are now

$$
h_{R R}=f_{R},
$$

and

yielding equilibrium if

$$
h_{A R}=\frac{1}{2}\left(f_{R}+f_{A}\right)-\frac{1}{2}\left|A_{A R}\right|\left(w_{R}-w_{A}\right)
$$

$$
\left(A_{A R}-\left|A_{A R}\right|\right)\left(w_{R}-w_{A}\right)=T(\Lambda-|\Lambda|) T^{-1}\left(w_{R}-w_{A}\right)=0 .
$$


With $u<c$ this is satisfied by the negative eigenvalue $u-c$, and since $w_{R}-w_{A}$ is the corresponding eigenvector, the Hugoniot equation

$$
f_{R}-f_{A}=S\left(w_{R}-w_{A}\right)
$$

is satisfied for the shock speed $S=u-c$. Thus $w_{A}$ again lies on a Hugoniot curve. At the entrance to the shock the transition from $w_{L}$ to $w_{A}$ is less than the full transition from $w_{L}$ to $w_{R}$ for which $u=c$. Thus a structure is admitted in which $u>c$ in the transition from $L$ to $A$, with the consequence that the flux is calculated from the upwind state

$$
f_{L A}=\frac{1}{2}\left(f_{A}+f_{L}\right)-\frac{1}{2} A_{L A}\left(w_{L}-w_{A}\right)=f_{L}
$$

and equilibrium is maintained.

\subsection{Case 3 Convective Upwind and Split Pressure (CUSP) Scheme}

Characteristic decomposition allows equilibrium to be established through full upwinding of the flux entering the transition layer, while the flux leaving the transition layer satisfies a Hugoniot equation. This can also be accomplished by a less complex scheme. Suppose that the diffusive flux is defined as

$$
d_{j+\frac{1}{2}}=\frac{1}{2} \alpha^{*} c\left(w_{j+1}-w_{j}\right)+\beta\left(f_{j+1}-f_{j}\right)
$$

where the factor $c$ is included so that $\alpha^{*}$ is dimensionless. Let $M$ be the Mach number $\frac{u}{c}$. If the flow is supersonic an upwind scheme is obtained by setting

$$
\alpha^{*}=0, \beta=\operatorname{sign}(M) \text {. }
$$

Introducing the Roe linearization, the Mach number is calculated from $u$ and $c$, and at the entrance to the shock a transition to an intermediate value $w_{\mathcal{A}}$ is admitted with $u>c$ and

$$
f_{L A}=\frac{1}{2}\left(f_{A}+f_{L}\right)-\frac{1}{2}\left(f_{A}-f_{L}\right)=f_{L}
$$

The fluxes leaving and entering the cell immediately to the right of the shock are now

$$
f_{R R}=f_{R},
$$

and

$$
f_{A R}=\frac{1}{2}\left(f_{R}+f_{A}\right)-\frac{1}{2} \alpha^{*} c\left(w_{R}-w_{A}\right)-\frac{1}{2} \beta\left(f_{R}-f_{A}\right) .
$$

These are in equilibrium if

$$
f_{R}-f_{A}+\frac{\alpha^{*} c}{1+\beta}\left(w_{R}-w_{A}\right)=0
$$

This is the Hugoniot equation for a shock moving to the left with a speed $\frac{\alpha^{*} c}{1+\beta}$. Also, introducing the Roe linearization,

$$
\left(A_{R A}+\frac{\alpha^{*} c}{1+\beta} I\right)\left(w_{R}-w_{A}\right)=0
$$

Thus $w_{R}-w_{A}$ is an eigenvector of $A_{R A}$ and $-\frac{\alpha^{*} c}{1+\beta}$ is the corresponding eigenvalue. Since the eigenvalues are $u, u+c$ and $u-c$, the only choice which leads to positive diffusion when $u>0$ is $u-c$, yielding the relationship

$$
\alpha^{*} c=(1+\beta)(c-u), 0<u<c .
$$

Thus $\beta$ is uniquely determined once $\alpha^{*}$ is chosen, leading to a one-parameter family of schemes. The choice $\beta=M$ corresponds to the Harten-Lax-Van Leer (HLL) scheme $[4,3]$, which is extremely diffusive.

The term $\beta\left(f_{R}-f_{A}\right)$ contributes to the diffusion of the convective terms. Suppose that the convective terms are separated by splitting the flux according to equations (8), (9) and (10). Then the total effective coefficient of convective diffusion is

$$
\alpha c=\alpha^{*} c+\beta \bar{u} .
$$


The choice $\alpha c=\bar{u}$ leads to low diffusion near a stagnation point, and also leads to a smooth continuation of convective diffusion across the sonic line since $\alpha^{*}=0$ and $\beta=1$ when $|M|>1$. The scheme must also be formulated so that the cases of $u>0$ and $u<0$ are treated symmetrically. Using the notation $M=\frac{u}{c}$, $\lambda^{ \pm}=u \pm c$, this leads to the diffusion coefficients

$$
\begin{gathered}
\alpha=|M| \\
\beta=\left\{\begin{array}{cc}
+\max \left(0, \frac{u+\lambda^{-}}{u-\lambda^{-}}\right) & \text {if } 0<M<1 \\
\left.-\max \left(0, \frac{u+\lambda^{+}}{u-\lambda^{+}}\right)\right) & \text {if }-1<M<0 \\
\operatorname{sign}(M) & \text { if }|M| \geq 1 .
\end{array}\right.
\end{gathered}
$$

Near a stagnation point $\alpha$ may be modified to $\alpha=\frac{1}{2}\left(\epsilon+\frac{|M|^{2}}{\epsilon}\right)$ if $|M|$ is smaller than a threshold $\epsilon$.

\subsubsection{Criteria for a single point shock}

The analysis of these three cases shows that a discrete shock structure with a single interior point is supported by artificial diffusion that satisfies the two conditions that

1. it produces an upwind flux if the flow is determined to be supersonic through the interface

2. it satisfies a generalized eigenvalue problem for the exit from the shock of the form

$$
\left(A_{A R}-\alpha_{A R} B_{A R}\right)\left(w_{R}-w_{A}\right)=0,
$$

where $A_{A R}$ is the linearized Jacobian matrix and $B_{A R}$ is the matrix defining the diffusion for the interface $A R$. These two conditions are satisfied by both the characteristic and CUSP schemes. Scalar diffusion does not satisfy the first condition.

\section{Schemes Admitting Constant Total Enthalpy in Steady Flow}

In steady flow the stagnation enthalpy $H$ is constant, corresponding to the fact that the energy and mass equations are consistent when the constant factor $H$ is removed from the energy equation. Discrete and semidiscrete schemes do not necessarily satisfy this property. In the case of a semi-discrete scheme expressed in viscosity form, equations (3) and (4), a solution with constant $H$ is admitted if the viscosity for the energy equation reduces to the viscosity for the continuity equation with $\rho$ replaced by $\rho H$. When the standard characteristic decomposition (6) is used, the viscous fluxes for $\rho$ and $\rho H$ which result from composition of the fluxes for the characteristic variables do not have this property, and $H$ is not constant in the discrete solution. In practice there is an excursion of $H$ in the discrete shock structure which represents a local heat source. In very high speed flows the corresponding error in the temperature may lead to a wrong prediction of associated effects such as chemical reactions.

The source of the error in the stagnation enthalpy is the discrepancy between the convective terms

$$
u\left(\begin{array}{c}
\rho \\
\rho u \\
\rho H
\end{array}\right),
$$

in the flux vector, which contain $\rho H$, and the state vector which contains $\rho E$. This may be remedied by introducing a modified state vector

$$
w_{h}=\left(\begin{array}{c}
\rho \\
\rho u \\
\rho H
\end{array}\right)
$$


Then one introduces the linearization

$$
f_{R}-f_{L}=A_{h}\left(w_{h_{R}}-w_{h_{L}}\right)
$$

Here $A_{h}$ may be calculated in the same way as the standard Roe linearization. On introducing the vector

$$
v=\left(\begin{array}{c}
\sqrt{\rho} \\
\sqrt{\rho} u \\
\sqrt{\rho} H
\end{array}\right)
$$

all quantities in both $f$ and $w_{h}$ are products of the form $v_{j} v_{k}$ which have the property that a finite difference $\Delta\left(v_{j} v_{k}\right)$ between left and right states can be expressed as

$$
\Delta\left(v_{j} v_{k}\right)=\overline{v_{j}} \Delta v_{k}+\overline{v_{k}} \Delta v_{j}
$$

where $\bar{v}_{j}$ is the arithmetic mean $\frac{1}{2}\left(v_{j R}+v_{j L}\right)$. Therefore,

$$
\Delta w=B \Delta v, \Delta f=C \Delta v=C B^{-1} \Delta w
$$

where $B$ and $C$ can be expressed in terms of appropriate mean values of the quantities $v_{j}$.

Define

$$
u=\frac{\sqrt{\rho_{R}} u_{R}+\sqrt{\rho_{L}} u_{L}}{\sqrt{\rho_{R}}+\sqrt{\rho_{L}}}, \quad H=\frac{\sqrt{\rho_{R}} H_{R}+\sqrt{\rho_{L}} H_{L}}{\sqrt{\rho_{R}}+\sqrt{\rho_{L}}}
$$

and

$$
c=\sqrt{(\gamma-1)\left(H-\frac{u^{2}}{2}\right)}
$$

Then

$$
A_{h}=\left(\begin{array}{ccc}
0 & 1 & 0 \\
-\frac{\gamma+1}{\gamma} \frac{u^{2}}{2} & \frac{\gamma+1}{\gamma} u & \frac{\gamma-1}{\gamma} \\
-u H & H & u
\end{array}\right) \text {. }
$$

The eigenvalues of $A_{h}$ are $u, \lambda^{+}$and $\lambda^{-}$where

$$
\lambda^{ \pm}=\frac{\gamma+1}{2 \gamma} u \pm \sqrt{\left(\frac{\gamma+1}{2 \gamma} u\right)^{2}+\frac{c^{2}-u^{2}}{\gamma}}
$$

Note that $\lambda^{+}$and $\lambda^{-}$have the same sign as $u+c$ and $u-c$, and change sign at the sonic line $u= \pm c$. The corresponding eigenvectors of $A_{h}$ are the columns of

$$
T=\left(\begin{array}{ccc}
1 & 1 & 1 \\
u & \lambda^{+} & \lambda^{-} \\
\frac{u^{2}}{2} & H & H
\end{array}\right)
$$

Also the left eigenvectors of $A_{h}$ are the rows of

$$
T^{-1}=\frac{1}{D}\left(\begin{array}{ccc}
\left(\lambda^{+}-\lambda^{-}\right) H & 0 & \left(\lambda^{+}-\lambda^{-}\right) \\
-\left(u H-\lambda^{-} \frac{u^{2}}{2}\right) & H-\frac{u^{2}}{2} & -\left(\lambda^{-}-u\right) \\
u H-\lambda^{+} \frac{u^{2}}{2} & -\left(H-\frac{u^{2}}{2}\right) & \lambda^{+}-u
\end{array}\right)
$$

where

$$
D=\left(\lambda^{+}-\lambda^{-}\right)\left(H-\frac{u^{2}}{2}\right)=\left(\lambda^{+}-\lambda^{-}\right) \frac{c^{2}}{\gamma-1} .
$$

Then

$$
A_{h}=T \Lambda T^{-1}
$$


where

$$
\Lambda=\left(\begin{array}{lll}
u & & \\
& \lambda^{+} & \\
& & \lambda^{-}
\end{array}\right) .
$$

The same development can be carried out for multidimensional flows. For convenience the formulas for the general multi-dimensional case are presented in the Appendix.

Using the modified linearization both the characteristic upwind scheme and the CUSP scheme can be reformulated as follows to admit steady solutions with constant $H$.

\subsection{Case 1 Characteristic Upwind Scheme}

The diffusion for the characteristic upwind scheme is now defined to be

$$
d_{j+\frac{1}{2}}=\frac{1}{2}\left|A_{h+\frac{1}{2}}\right|\left(w_{j+1}-w j\right),
$$

in which $\left|A_{h}\right|$ is defined to be

$$
\left|A_{h}\right|=T \Lambda T^{-1}
$$

where $T$ is the eigenvector matrix of $A_{h}$, and

$$
|\Lambda|=\left(\begin{array}{lll}
|u| & & \\
& \left|\lambda^{+}\right| & \\
& & \left|\lambda^{-}\right|
\end{array}\right) .
$$

In order to show that the scheme admits a solution with constant $H$, split the diffusion into two parts

$$
d_{j+\frac{1}{2}}=d_{j+\frac{1}{2}}^{(1)}+d_{j+\frac{1}{2}}^{(2)}
$$

where $d_{j+\frac{1}{2}}^{(1)}$ is the contribution from $|u|$, and where $d_{j+\frac{1}{2}}^{(2)}$ is the contribution from $\left|\lambda^{+}\right|$and $\left|\lambda^{-}\right|$. Then

$$
\begin{aligned}
d_{j+\frac{1}{2}}^{(2)} & =\left(\begin{array}{ccc}
1 & 1 & 1 \\
u & \lambda^{+} & \lambda^{-} \\
\frac{u^{2}}{2} & H & H
\end{array}\right)\left(\begin{array}{ccc}
0 & & \\
& \left|\lambda^{+}\right| & \\
& & \left|\lambda^{-}\right|
\end{array}\right) T^{-1} \Delta w_{h} \\
& =\left(\begin{array}{ccc}
0 & \left|\lambda^{+}\right| & \left|\lambda^{-}\right| \\
0 & \left|\lambda^{+}\right| \lambda^{+} & \left|\lambda^{-}\right| \lambda^{-} \\
0 & \left|\lambda^{+}\right| H & \left|\lambda^{-}\right| H
\end{array}\right) T^{-1} \Delta w_{h}
\end{aligned}
$$

and the third element of $d_{j+\frac{1}{2}}^{(2)}$ equals the first element multiplied by $H$. Also

$$
\begin{aligned}
d_{j+\frac{1}{2}}^{(1)} & =\frac{(\gamma-1)|u|}{c^{2}}\left(\begin{array}{ccc}
1 & 1 & 1 \\
u & \lambda^{+} & \lambda^{-} \\
\frac{u^{2}}{2} & H & H
\end{array}\right)\left(\begin{array}{c}
H \Delta \rho-\Delta(\rho H) \\
0 \\
0
\end{array}\right) \\
& =\frac{(\gamma-1) \rho|u| \Delta H}{c^{2}}\left(\begin{array}{c}
1 \\
u \\
\frac{u^{2}}{2}
\end{array}\right)
\end{aligned}
$$

and this is zero if $H$ is constant. Thus both contributions are consistent with a steady solution in which $H$ is constant. The two variations of the characteristic splitting can conveniently be distinguished as the $\mathrm{E}$ and H-characteristic schemes. 


\subsection{Case 2 CUSP Scheme}

The diffusive flux is now expressed as

$$
d_{j+\frac{1}{2}}=\frac{1}{2} \alpha^{\prime \prime} c \Delta w_{h}+\frac{1}{2} \beta \Delta f,
$$

where $\Delta$ denotes the difference from $j+1$ to $j$. Again equilibrium at the entrance is established by upwinding, while equilibrium at the exit requires

$$
\Delta f+\frac{\alpha^{*} c}{1+\beta} \Delta w_{h}=\left(A_{h}+\frac{\alpha^{*} c}{1+\beta} l\right) \Delta w_{h}=0 .
$$

Therefore, $-\frac{\alpha^{*} c}{1+\beta}$ must be an eigenvalue of $A_{h}$, and in the case $u>0$, positive diffusion is obtained by taking

$$
\alpha^{*} c=-(1+\beta) \lambda^{-} \text {. }
$$

Now the split is redefined as

$$
f=u w_{h}+f_{p}
$$

where

$$
f_{p}=\left(\begin{array}{l}
0 \\
p \\
0
\end{array}\right)
$$

and the diffusive flux can be expressed as

$$
d_{j+\frac{1}{2}}=\frac{1}{2} \alpha c \Delta w_{h}+\beta \bar{w}_{h} \Delta u+\beta \Delta f_{p}
$$

Then $\alpha$ and $\beta$ are defined as before by equations (19) and (20), using the modified eigenvalues $\lambda^{ \pm}$defined equation (21). This splitting corresponds to the Liou-Steffen splitting $[7,13]$. The splitting in which the convective terms contain $\rho E$ corresponds to the wave particle splitting $[8,2]$. As in the case of characteristic splitting, the two variations can conveniently be distinguished as the E-CUSP and H-CUSP schemes.

\section{Implementation of limiters for the CUSP scheme}

In the case of a scalar conservation law, high resolution schemes which guarantee the preservation of the positivity or monotonicity of the solution can be constructed by limiting the action of higher order or antidiffusive terms, which might otherwise cause extrema to grow. Typically, these schemes, such as both the symmetric and upstream limited positive (SLIP and USLIP) schemes discussed in the previous paper in this series [5], compare the slope of the solution at nearby mesh intervals. The characteristic upwind scheme essentially applies the same construction to the characteristic variables, so that the solution is subject to controls on the formulation or growth of extrema of these variables. The fluxes appearing in the CUSP scheme have different slopes approaching from either side of the sonic line, and use of limiters which depends on comparisons of the slopes of these fluxes can lead to a loss of smoothness in the solution at the entrance to supersonic zones in the flow.

An alternative formulation which avoids this difficulty, and may be used with either the characteristic upwind or the CUSP scheme, is to form the diffusive flux from left and right states at the cell interface. These are interpolated or extrapolated from nearby data, subject to limiters to preserve monotonicity, in a similar manner to the reconstruction of the solution in Van Leer's MUSCL scheme [6]. Let

$$
R(u, v)=1-\left|\frac{u-v}{|u|+|v|}\right|^{q}
$$

where $q$ is a positive power. Then $R(u, v)=0$ when $u$ and $v$ have opposite sign. Also define

$$
L(u, v)=\frac{1}{2} R(u, v)(u+v)
$$


Let $w^{(k)}$ denote the $k$ th element of the state vector $w$. Now define left and right states for each dependent variable separately as

$$
\begin{aligned}
& w_{L}^{(k)}=w_{j}^{(k)}+\frac{1}{2} L\left(\Delta w_{j+\frac{3}{2}}^{(k)}, \Delta w_{j-\frac{1}{2}}^{(k)}\right) \\
& w_{R}^{(k)}=w_{j+1}^{(k)}-\frac{1}{2} L\left(\Delta w_{j+\frac{3}{2}}^{(k)}, \Delta w_{j-\frac{1}{2}}^{(k)}\right)
\end{aligned}
$$

where

$$
\Delta w_{j+\frac{1}{2}}=w_{j+1}-w_{j}
$$

Then

$$
w_{R}^{(k)}-w_{L}^{(k)}=\Delta w_{j+\frac{1}{2}}^{(k)}-L\left(\Delta w_{j+\frac{3}{2}}^{(k)}, \Delta w_{j-\frac{1}{2}}^{(k)}\right)
$$

and in the case of a scalar equation the SLIP scheme [5] is recovered by making the diffusive flux proportional to this difference. To implement the CUSP scheme the pressures $p_{L}$ and $p_{R}$ for the left and right states are determined from $w_{L}$ and $w_{R}$. Then the diffusive flux is calculated by substituting $w_{L}$ for $w_{j}$ and $w_{R}$ for $w_{j+1}$ to give

$$
d_{j+\frac{1}{2}}=\frac{1}{2} \alpha^{*} c\left(w_{R}-w_{L}\right)+\frac{1}{2} \beta\left(f\left(w_{R}\right)-f\left(w_{L}\right)\right) .
$$

Similarly the characteristic upwind scheme is implemented by calculating $A_{j+\frac{1}{2}}$ from $w_{R}$ and $w_{L}$. An alternative reconstruction is to set

$$
\begin{aligned}
& w_{L}^{(k)}=w_{j}^{(k)}+R\left(\Delta w_{j+\frac{3}{2}}^{(k)}, \Delta w_{j-\frac{1}{2}}^{(k)}\right) \Delta w_{j-\frac{1}{2}}^{(k)} \\
& w_{R}^{(k)}=w_{j}^{(k)}-R\left(\Delta w_{j+\frac{3}{2}}^{(k)}, \Delta w_{j-\frac{1}{2}}^{(k)}\right) \Delta w_{j+\frac{3}{2}}^{(k)} .
\end{aligned}
$$

It has been found that essentially similar results are obtained in numerical calculations of steady flows using the two interpolation formulas.

\section{$7 \quad$ Numerical Results}

Extensive numerical tests have been performed with the $\mathrm{E}$ and $\mathrm{H}$-characteristic and the $\mathrm{E}$ and $\mathrm{H}$-CUSP schemes to verify their properties. Results for one, two and three-dimensional flows are presented in sections $7.1,7.2$ and 7.3 .

\subsection{One dimensional shock}

In order to verify the discrete structure of stationary shocks with the various schemes, calculations were performed for a one dimensional problem with initial data containing left and right states compatible with the Rankine-Hugoniot conditions. An intermediate state consisting of the arithmetic average of the left and right states was introduced at a single cell in the center of the domain. With this intermediate state the system is not in equilibrium, and the time dependent equations were solved to find an equilibrium solution with a stationary shock wave separating the left and right states. Tables 1 through 4 shows the results for a shock wave at Mach 20 for the E-characteristic, H-characteristic, E-CUSP and H-CUSP schemes. In all cases the SLIP construction was used with the limiter defined by equations (22) and (23), and $q=3$. The tables show the values of $\rho, u, H, p, M$ and the entropy $S=\log \frac{p}{\rho \gamma}-\log \left(\frac{p_{L}}{\rho_{L}}\right)$. All four schemes display a perfect one point shock structure. The entropy is zero to 4 decimal places upstream of the shock, and is constant to 4 decimal places downstream of the shock. There is a slight excursion of the entropy at the interior point in the results for the $\mathrm{H}$-characteristic and H-CUSP schemes. Correspondingly there is an excursion in the stagnation enthalpy at the interior point in the results for the E-characteristic and E-CUSP schemes. It may be noted that the mass, momentum and energy of the initial data are not compatible with the final equilibrium state. According to equation (12) the total mass, momentum and energy must remain constant if the outflow flux $f_{R}$ remains equal to the inflow flux $f_{L}$. Therefore $f_{R}$ must be allowed to vary according to an appropriate outflow 
boundary condition to allow the total mass, momentum and energy to be adjusted to values compatible with equilibrium.

\subsection{Airfoil calculations}

The results of transonic flow calculations using the H-characteristic and H-CUSP schemes are compared in figures (2-8). The E-characteristic and E-CUSP schemes produce results which are very similar to the results of the H-characteristic and H-CUSP schemes, with small deviations in stagnation enthalpy. These are eliminated by the H-characteristic and H-CUSP schemes. The limiter defined by equations (22) and (23) was again used with $q=3$ in both schemes to define left and right states in the manner described in section 6 . The H-CUSP scheme was simplified by replacing the Roe averages (2) by arithmetic averages, and using $\lambda^{ \pm}=u \pm c$ in the formula (20) for $\beta$. It was also found that the term $\bar{w}_{h} \Delta u$ tends to reduce the rate of convergence to a steady

state. Therefore it was attenuated by the factor $\frac{\left|p_{R}-p_{L}\right|}{\left(\left|p_{R}-p_{S}\right|+\left|p_{L}-p_{S}\right|\right.}$ where $p_{S}$ is the pressure at sonic speed, and $p_{L}$ and $p_{R}$ are the pressures to the left and right. When the flow crosses the sonic line $p_{S}$ lies between $p_{L}$ and $p_{R}$, and this factor becomes unity. Thus the full scheme is restored at a shock wave. All the calculations were performed with the five stage modified Runge-Kutta time stepping scheme described in reference [5]. Convergence to a steady state was accelerated by the multigrid also described in reference [5], using W-cycles in which a single time step is performed on each grid level during the descent towards coarser grids. The total amount of work in each W-cycle is about the same as two time steps on the fine grid.

Calculations are presented for two well known airfoils, the RAE 2822 and the NACA 0012. The equations were discretized on meshes with $\mathrm{O}$-topology extending out to a radius of about 100 chords. In each case the calculations were performed on a sequence of successively finer meshes from $40 \times 8$ to $320 \times 64$ cells, while the multigrid cycles on each of these meshes descended to a coarsest mesh of $10 \times 2$ cells. Figure 2 shows the inner parts of the 160x32 meshes for the two airfoils. Figures 3-8 show the final results for each scheme on $320 \times 64$ meshes for the RAE 2822 airfoil at Mach .75 and $3^{\circ}$ angle of attack, and for the NACA 0012 airfoil at Mach .8 and $1.25^{\circ}$ angle of attack, and also at Mach .85 and $1^{\circ}$ angle of attack. In each case the convergence history is shown for 100 or 200 cycles, while the pressure distribution is displayed after a sufficient number of cycles for its convergence. The pressure distribution of the RAE 2822 airfoil converged in only 25 cycles. Convergence was slower for the NACA 0012 airfoil. In the case of flow at Mach .8 and $1.25^{\circ}$ angle of attack, additional cycles were needed to damp out a wave downstream of the weak shock wave on the lower surface.

As a further check on accuracy the drag coefficient should be zero in subsonic flow, or in shock free transonic flow. Tables 5 and 6 show the computed drag coefficient with the H-characteristic and H-CUSP schemes on a sequence of three meshes for three examples. The first two are subsonic flows over the RAE 2822 and NACA 0012 airfoils at Mach .5 and $3^{\circ}$ angle of attack. The third is the flow over the shock free Korn airfoil at its design point of Mach .75 and $0^{\circ}$ angle of attack. The computed drag coefficients are slightly lower with the $\mathrm{H}$-CUSP scheme: in all three cases the drag coefficient is calculated to be zero to four digits on a $160 \times 32 \mathrm{mesh}$.

\subsection{Three-dimensional calculations for a swept wing}

As a further test of the performance of the H-CUSP scheme, the flow past the ONERA M6 wing was calculated on a mesh with $\mathrm{C}-\mathrm{H}$ topology and $192 \times 32 \times 48=294912$ cells. Figure 9 shows the result at Mach .84 and $3.06^{\circ}$ angle of attack. This again verifies the non-oscillatory character of the solution, and the sharp resolution of shock waves. In this case 50 cycles were sufficient for convergence of the pressure distributions.

\section{Conclusion}

It was shown in the first paper in this series [5] that the concept of local extremum diminishing (LED) schemes provides a convenient framework for the formulation of non-oscillatory shock capturing schemes for 


\begin{tabular}{|c|c|c|c|c|c|c|}
\hline I & $\rho$ & $\mathbf{u}$ & $\mathrm{H}$ & $\mathrm{p}$ & M & $\mathbf{s}$ \\
\hline 1 & 1.0000 & 23.6643 & 283.5000 & 1.0000 & 20.0000 & 0.0000 \\
\hline 2 & 1.0000 & 23.6643 & 283.5000 & 1.0000 & 20.0000 & 0.0000 \\
\hline 3 & 1.0000 & 23.6643 & 283.5000 & 1.0000 & 20.0000 & 0.0000 \\
\hline 4 & 1.0000 & 23.6643 & 283.5000 & 1.0000 & 20.0000 & 0.0000 \\
\hline 5 & 1.0000 & 23.6643 & 283.5000 & 1.0000 & 20.0000 & 0.0000 \\
\hline 6 & 1.0000 & 23.6643 & 283.5000 & 1.0000 & 20.0000 & 0.0000 \\
\hline 7 & 1.0000 & 23.6643 & 283.5000 & 1.0000 & 20.0000 & 0.0000 \\
\hline 8 & 1.0000 & 23.6643 & 283.5000 & 1.0000 & 20.0000 & 0.0000 \\
\hline 9 & 1.0000 & 23.6643 & 283.5000 & 1.0000 & 20.0000 & 0.0000 \\
\hline 10 & 1.0000 & 23.6643 & 283.5000 & 1.0000 & 20.0000 & 0.0000 \\
\hline 11 & 1.0000 & 23.6643 & 283.5000 & 1.0000 & 20.0000 & 0.0000 \\
\hline 12 & 1.0000 & 23.6643 & 283.5000 & 1.0000 & 20.0000 & 0.0000 \\
\hline 13 & 1.0000 & 23.6643 & 283.5000 & 1.0000 & 20.0000 & 0.0000 \\
\hline 14 & 1.0000 & 23.6643 & 283.5000 & 1.0000 & 20.0000 & 0.0000 \\
\hline 15 & 1.0000 & 23.6643 & 283.5000 & 1.0000 & 20.0000 & 0.0000 \\
\hline 16 & 1.0000 & 23.6643 & 283.5000 & 1.0000 & 20.0000 & 0.0000 \\
\hline 17 & 1.0000 & 23.6643 & 283.5000 & 1.0000 & 20.0000 & 0.0000 \\
\hline 18 & 1.0000 & 23.6643 & 283.5000 & 1.0000 & 20.0000 & 0.0000 \\
\hline 19 & 1.0000 & 23.6643 & 283.5000 & 1.0000 & 20.0000 & 0.0000 \\
\hline 20 & 1.0000 & 23.6643 & 283.5000 & 1.0000 & 20.0000 & 0.0000 \\
\hline 21 & 1.0000 & 23.6643 & 283.5000 & 1.0000 & 20.0000 & 0.0000 \\
\hline 22 & 4.4232 & 6.9992 & 268.9099 & 308.8882 & 0.7079 & 37.5269 \\
\hline 23 & 5.9259 & 3.9930 & 283.5064 & 466.5228 & 0.3803 & 37.6369 \\
\hline 24 & 5.9260 & 3.9930 & 283.5064 & 466.5228 & 0.3803 & 37.6369 \\
\hline 25 & 5.9260 & 3.9930 & 283.5064 & 466.5228 & 0.3803 & 37.6369 \\
\hline 26 & 5.9260 & 3.9930 & 283.5064 & 466.5228 & 0.3803 & 37.6369 \\
\hline 27 & 5.9260 & 3.9930 & 283.5064 & 466.5228 & 0.3803 & 37.6369 \\
\hline 28 & 5.9260 & 3.9930 & 283.5064 & 466.5228 & 0.3803 & 37.6369 \\
\hline 29 & 5.9260 & 3.9930 & 283.5064 & 466.5228 & 0.3803 & 37.6369 \\
\hline 30 & 5.9260 & 3.9930 & 283.5064 & 466.5228 & 0.3803 & 37.6369 \\
\hline 31 & 5.9260 & 3.9930 & 283.5064 & 466.5228 & 0.3803 & 37.6369 \\
\hline 32 & 5.9260 & 3.9930 & 283.5064 & 466.5228 & 0.3803 & 37.6369 \\
\hline 33 & 5.9260 & 3.9930 & 283.5064 & 466.5228 & 0.3803 & 37.6369 \\
\hline 34 & 5.9260 & 3.9930 & 283.5064 & 466.5228 & 0.3803 & 37.6369 \\
\hline 35 & 5.9260 & 3.9930 & 283.5064 & 466.5228 & 0.3803 & 37.6369 \\
\hline 36 & 5.9260 & 3.9930 & 283.5064 & 466.5228 & 0.3803 & 37.6369 \\
\hline 37 & 5.9260 & 3.9930 & 283.5064 & 466.5228 & 0.3803 & 37.6369 \\
\hline 38 & 5.9260 & 3.9930 & 283.5064 & 466.5228 & 0.3803 & 37.6369 \\
\hline 39 & 5.9260 & 3.9930 & 283.5064 & 466.5228 & 0.3803 & 37.6369 \\
\hline 40 & 5.9260 & 3.9930 & 283.5064 & 466.5228 & 0.3803 & 37.6369 \\
\hline 41 & 5.9260 & 3.9930 & 283.5064 & 466.5228 & 0.3803 & 37.6369 \\
\hline 42 & 5.9260 & 3.9930 & 283.5064 & 466.5228 & 0.3803 & 37.6369 \\
\hline
\end{tabular}

Table 1: Shock Wave at Mach 20: E-characteristic scheme 


\begin{tabular}{|c|c|c|c|c|c|c|}
\hline$I$ & $\rho$ & $\mathbf{u}$ & $\mathrm{H}$ & p & $\bar{M}$ & $\bar{s}$ \\
\hline 1 & 1.0000 & 23.6643 & 283.5000 & 1.0000 & 20.0000 & 0.0000 \\
\hline 2 & 1.0000 & 23.6643 & 283.5000 & 1.0000 & 20.0000 & 0.0000 \\
\hline 3 & 1.0000 & 23.6643 & 283.5000 & 1.0000 & 20.0000 & 0.0000 \\
\hline 4 & 1.0000 & 23.6643 & 283.5000 & 1.0000 & 20.0000 & 0.0000 \\
\hline 5 & 1.0000 & 23.6643 & 283.5000 & 1.0000 & 20.0000 & 0.0000 \\
\hline 6 & 1.0000 & 23.6643 & 283.5000 & 1.0000 & 20.0000 & 0.0000 \\
\hline 7 & 1.0000 & 23.6643 & 283.5000 & 1.0000 & 20.0000 & 0.0000 \\
\hline 8 & 1.0000 & 23.6643 & 283.5000 & 1.0000 & 20.0000 & 0.0000 \\
\hline 9 & 1.0000 & 23.6643 & 283.5000 & 1.0000 & 20.0000 & 0.0000 \\
\hline 10 & 1.0000 & 23.6643 & 283.5000 & 1.0000 & 20.0000 & 0.0000 \\
\hline 11 & 1.0000 & 23.6643 & 283.5000 & 1.0000 & 20.0000 & 0.0000 \\
\hline 12 & 1.0000 & 23.6643 & 283.5000 & 1.0000 & 20.0000 & 0.0000 \\
\hline 13 & 1.0000 & 23.6643 & 283.5000 & 1.0000 & 20.0000 & 0.0000 \\
\hline 14 & 1.0000 & 23.6643 & 283.5000 & 1.0000 & 20.0000 & 0.0000 \\
\hline 15 & 1.0000 & 23.6643 & 283.5000 & 1.0000 & 20.0000 & 0.0000 \\
\hline 16 & 1.0000 & 23.6643 & 283.5000 & 1.0000 & 20.0000 & 0.0000 \\
\hline 17 & 1.0000 & 23.6643 & 283.5000 & 1.0000 & 20.0000 & 0.0000 \\
\hline 18 & 1.0000 & 23.6643 & 283.5000 & 1.0000 & 20.0000 & 0.0000 \\
\hline 19 & 1.0000 & 23.6643 & 283.5000 & 1.0000 & 20.0000 & 0.0000 \\
\hline 20 & 1.0000 & 23.6643 & 283.5000 & 1.0000 & 20.0000 & 0.0000 \\
\hline 21 & 1.0000 & 23.6643 & 283.5000 & 1.0000 & 20.0000 & 0.0000 \\
\hline 22 & 4.2476 & 7.1962 & 283.5073 & 312.6405 & 0.7089 & 40.2710 \\
\hline 23 & 5.9259 & 3.9930 & 283.5073 & 466.5208 & 0.3803 & 37.6371 \\
\hline 24 & 5.9260 & 3.9930 & 283.5073 & 466.5208 & 0.3803 & 37.6371 \\
\hline 25 & 5.9260 & 3.9930 & 283.5073 & 466.5208 & 0.3803 & 37.6371 \\
\hline 26 & 5.9260 & 3.9930 & 283.5073 & 466.5208 & 0.3803 & 37.6371 \\
\hline 27 & 5.9260 & 3.9930 & 283.5073 & 466.5208 & 0.3803 & 37.6371 \\
\hline 28 & 5.9260 & 3.9930 & 283.5073 & 466.5208 & 0.3803 & 37.6371 \\
\hline 29 & 5.9260 & 3.9930 & 283.5073 & 466.5208 & 0.3803 & 37.6371 \\
\hline 30 & 5.9260 & 3.9930 & 283.5073 & 466.5208 & 0.3803 & 37.6371 \\
\hline 31 & 5.9260 & 3.9930 & 283.5074 & 466.5208 & 0.3803 & 37.6371 \\
\hline 32 & 5.9260 & 3.9930 & 283.5074 & 466.5208 & 0.3803 & 37.6371 \\
\hline 33 & 5.9260 & 3.9930 & 283.5074 & 466.5208 & 0.3803 & 37.6371 \\
\hline 34 & 5.9260 & 3.9930 & 283.5074 & 466.5208 & 0.3803 & 37.6371 \\
\hline 35 & 5.9260 & 3.9930 & 283.5074 & 466.5208 & 0.3803 & 37.6371 \\
\hline 36 & 5.9260 & 3.9930 & 283.5074 & 466.5208 & 0.3803 & 37.6371 \\
\hline 37 & 5.9260 & 3.9930 & 283.5073 & 466.5208 & 0.3803 & 37.6371 \\
\hline 38 & 5.9260 & 3.9930 & 283.5073 & 466.5208 & 0.3803 & 37.6371 \\
\hline 39 & 5.9260 & 3.9930 & 283.5073 & 466.5208 & 0.3803 & 37.6371 \\
\hline 40 & 5.9260 & 3.9930 & 283.5073 & 466.5208 & 0.3803 & 37.6371 \\
\hline 41 & 5.9260 & 3.9930 & 283.5074 & 466.5208 & 0.3803 & 37.6371 \\
\hline 42 & 5.9260 & 3.9930 & 283.5074 & 466.5208 & 0.3803 & 37.6371 \\
\hline
\end{tabular}

Table 2: Shock Wave at Mach 20: H-characteristic scheme 


\begin{tabular}{|c|c|c|c|c|c|c|}
\hline I & $\rho$ & $\mathbf{u}$ & $\mathrm{H}$ & $\mathbf{p}$ & $\mathbf{M}$ & $\mathbf{s}$ \\
\hline 1 & 1.0000 & 23.6643 & 283.5000 & 1.0000 & 20.0000 & 0.0000 \\
\hline 2 & 1.0000 & 23.6643 & 283.5000 & 1.0000 & 20.0000 & 0.0000 \\
\hline 3 & 1.0000 & 23.6643 & 283.5000 & 1.0000 & 20.0000 & 0.0000 \\
\hline 4 & 1.0000 & 23.6643 & 283.5000 & 1.0000 & 20.0000 & 0.0000 \\
\hline 5 & 1.0000 & 23.6643 & 283.5000 & 1.0000 & 20.0000 & 0.0000 \\
\hline 6 & 1.0000 & 23.6643 & 283.5000 & 1.0000 & 20.0000 & 0.0000 \\
\hline 7 & 1.0000 & 23.6643 & 283.5000 & 1.0000 & 20.0000 & 0.0000 \\
\hline 8 & 1.0000 & 23.6643 & 283.5000 & 1.0000 & 20.0000 & 0.0000 \\
\hline 9 & 1.0000 & 23.6643 & 283.5000 & 1.0000 & 20.0000 & 0.0000 \\
\hline 10 & 1.0000 & 23.6643 & 283.5000 & 1.0000 & 20.0000 & 0.0000 \\
\hline 11 & 1.0000 & 23.6643 & 283.5000 & 1.0000 & 20.0000 & 0.0000 \\
\hline 12 & 1.0000 & 23.6643 & 283.5000 & 1.0000 & 20.0000 & 0.0000 \\
\hline 13 & 1.0000 & 23.6643 & 283.5000 & 1.0000 & 20.0000 & 0.0000 \\
\hline 14 & 1.0000 & 23.6643 & 283.5000 & 1.0000 & 20.0000 & 0.0000 \\
\hline 15 & 1.0000 & 23.6643 & 283.5000 & 1.0000 & 20.0000 & 0.0000 \\
\hline 16 & 1.0000 & 23.6643 & 283.5000 & 1.0000 & 20.0000 & 0.0000 \\
\hline 17 & 1.0000 & 23.6643 & 283.5000 & 1.0000 & 20.0000 & 0.0000 \\
\hline 18 & 1.0000 & 23.6643 & 283.5000 & 1.0000 & 20.0000 & 0.0000 \\
\hline 19 & 1.0000 & 23.6643 & 283.5000 & 1.0000 & 20.0000 & 0.0000 \\
\hline 20 & 1.0000 & 23.6643 & 283.5000 & 1.0000 & 20.0000 & 0.0000 \\
\hline 21 & 1.0000 & 23.6643 & 283.5000 & 1.0000 & 20.0000 & 0.0000 \\
\hline 22 & 4.4011 & 7.0503 & 268.7344 & 306.6689 & 0.7138 & 37.5200 \\
\hline 23 & 5.9259 & 3.9935 & 283.4970 & 466.4894 & 0.3804 & 37.6357 \\
\hline 24 & 5.9259 & 3.9935 & 283.4970 & 466.4894 & 0.3804 & 37.6357 \\
\hline 25 & 5.9259 & 3.9935 & 283.4970 & 466.4894 & 0.3804 & 37.6357 \\
\hline 26 & 5.9259 & 3.9935 & 283.4970 & 466.4894 & 0.3804 & 37.6357 \\
\hline 27 & 5.9259 & 3.9935 & 283.4970 & 466.4894 & 0.3804 & 37.6357 \\
\hline 28 & 5.9259 & 3.9935 & 283.4970 & 466.4894 & 0.3804 & 37.6357 \\
\hline 29 & 5.9259 & 3.9935 & 283.4970 & 466.4894 & 0.3804 & 37.6357 \\
\hline 30 & 5.9259 & 3.9935 & 283.4970 & 466.4894 & 0.3804 & 37.6357 \\
\hline 31 & 5.9259 & 3.9935 & 283.4970 & 466.4894 & 0.3804 & 37.6357 \\
\hline 32 & 5.9259 & 3.9935 & 283.4970 & 466.4894 & 0.3804 & 37.6357 \\
\hline 33 & 5.9259 & 3.9935 & 283.4970 & 466.4894 & 0.3804 & 37.6357 \\
\hline 34 & 5.9259 & 3.9935 & 283.4970 & 466.4894 & 0.3804 & 37.6357 \\
\hline 35 & 5.9259 & 3.9935 & 283.4970 & 466.4894 & 0.3804 & 37.6357 \\
\hline 36 & 5.9259 & 3.9935 & 283.4970 & 466.4894 & 0.3804 & 37.6357 \\
\hline 37 & 5.9259 & 3.9935 & 283.4970 & 466.4894 & 0.3804 & 37.6357 \\
\hline 38 & 5.9259 & 3.9935 & 283.4970 & 466.4894 & 0.3804 & 37.6357 \\
\hline 39 & 5.9259 & 3.9935 & 283.4970 & 466.4894 & 0.3804 & 37.6357 \\
\hline 40 & 5.9259 & 3.9935 & 283.4970 & 466.4894 & 0.3804 & 37.6357 \\
\hline 41 & 5.9259 & 3.9935 & 283.4970 & 466.4894 & 0.3804 & 37.6357 \\
\hline 42 & 5.9259 & 3.9935 & 283.4970 & 466.4894 & 0.3804 & 37.6357 \\
\hline
\end{tabular}

Table 3: Shock Wave at Mach 20: E-CUSP scheme 


\begin{tabular}{|c|c|c|c|c|c|c|}
\hline I & $\rho$ & $u$ & $\mathrm{H}$ & $\mathrm{p}$ & $\mathrm{M}$ & $\mathbf{s}$ \\
\hline 1 & 1.0000 & 23.6643 & 283.5000 & 1.0000 & 20.0000 & 0.0000 \\
\hline 2 & 1.0000 & 23.6643 & 283.5000 & 1.0000 & 20.0000 & 0.0000 \\
\hline 3 & 1.0000 & 23.6643 & 283.5000 & 1.0000 & 20.0000 & 0.0000 \\
\hline 4 & 1.0000 & 23.6643 & 283.5000 & 1.0000 & 20.0000 & 0.0000 \\
\hline 5 & 1.0000 & 23.6643 & 283.5000 & 1.0000 & 20.0000 & 0.0000 \\
\hline 6 & 1.0000 & 23.6643 & 283.5000 & 1.0000 & 20.0000 & 0.0000 \\
\hline 7 & 1.0000 & 23.6643 & 283.5000 & 1.0000 & 20.0000 & 0.0000 \\
\hline 8 & 1.0000 & 23.6643 & 283.5000 & 1.0000 & 20.0000 & 0.0000 \\
\hline 9 & 1.0000 & 23.6643 & 283.5000 & 1.0000 & 20.0000 & 0.0000 \\
\hline 10 & 1.0000 & 23.6643 & 283.5000 & 1.0000 & 20.0000 & 0.0000 \\
\hline 11 & 1.0000 & 23.6643 & 283.5000 & 1.0000 & 20.0000 & 0.0000 \\
\hline 12 & 1.0000 & 23.6643 & 283.5000 & 1.0000 & 20.0000 & 0.0000 \\
\hline 13 & 1.0000 & 23.6643 & 283.5000 & 1.0000 & 20.0000 & 0.0000 \\
\hline 14 & 1.0000 & 23.6643 & 283.5000 & 1.0000 & 20.0000 & 0.0000 \\
\hline 15 & 1.0000 & 23.6643 & 283.5000 & 1.0000 & 20.0000 & 0.0000 \\
\hline 16 & 1.0000 & 23.6643 & 283.5000 & 1.0000 & 20.0000 & 0.0000 \\
\hline 17 & 1.0000 & 23.6643 & 283.5000 & 1.0000 & 20.0000 & 0.0000 \\
\hline 18 & 1.0000 & 23.6643 & 283.5000 & 1.0000 & 20.0000 & 0.0000 \\
\hline 19 & 1.0000 & 23.6643 & 283.5000 & 1.0000 & 20.0000 & 0.0000 \\
\hline 20 & 1.0000 & 23.6643 & 283.5000 & 1.0000 & 20.0000 & 0.0000 \\
\hline 21 & 1.0000 & 23.6643 & 283.5000 & 1.0000 & 20.0000 & 0.0000 \\
\hline 22 & 4.1924 & 7.3248 & 283.4960 & 307.4467 & 0.7229 & 40.3353 \\
\hline 23 & 5.9259 & 3.9935 & 283.4960 & 466.4889 & 0.3804 & 37.6355 \\
\hline 24 & 5.9259 & 3.9935 & 283.4960 & 466.4889 & 0.3804 & 37.6355 \\
\hline 25 & 5.9259 & 3.9935 & 283.4960 & 466.4889 & 0.3804 & 37.6355 \\
\hline 26 & 5.9259 & 3.9935 & 283.4960 & 466.4889 & 0.3804 & 37.6355 \\
\hline 27 & 5.9259 & 3.9935 & 283.4960 & 466.4889 & 0.3804 & 37.6355 \\
\hline 28 & 5.9259 & 3.9935 & 283.4960 & 466.4889 & 0.3804 & 37.6355 \\
\hline 29 & 5.9259 & 3.9935 & 283.4960 & 466.4889 & 0.3804 & 37.6355 \\
\hline 30 & 5.9259 & 3.9935 & 283.4960 & 466.4889 & 0.3804 & 37.6355 \\
\hline 31 & 5.9259 & 3.9935 & 283.4960 & 466.4889 & 0.3804 & 37.6355 \\
\hline 32 & 5.9259 & 3.9935 & 283.4960 & 466.4889 & 0.3804 & 37.6355 \\
\hline 33 & 5.9259 & 3.9935 & 283.4960 & 466.4889 & 0.3804 & 37.6355 \\
\hline 34 & 5.9259 & 3.9935 & 283.4960 & 466.4889 & 0.3804 & 37.6355 \\
\hline 35 & 5.9259 & 3.9935 & 283.4960 & 466.4889 & 0.3804 & 37.6355 \\
\hline 36 & 5.9259 & 3.9935 & 283.4960 & 466.4889 & 0.3804 & 37.6355 \\
\hline 37 & 5.9259 & 3.9935 & 283.4960 & 466.4889 & 0.3804 & 37.6355 \\
\hline 38 & 5.9259 & 3.9935 & 283.4960 & 466.4889 & 0.3804 & 37.6355 \\
\hline 39 & 5.9259 & 3.9935 & 283.4960 & 466.4889 & 0.3804 & 37.6355 \\
\hline 40 & 5.9259 & 3.9935 & 283.4961 & 466.4889 & 0.3804 & 37.6355 \\
\hline 41 & 5.9259 & 3.9935 & 283.4961 & 466.4889 & 0.3804 & 37.6355 \\
\hline 42 & 5.9259 & 3.9935 & 283.4961 & 466.4889 & 0.3804 & 37.6355 \\
\hline
\end{tabular}

Table 4: Shock Wave at Mach 20: H-CUSP scheme

\begin{tabular}{|r|rrr|}
\hline Mesh & RAE 2822 & NACA 0012 & Korn Airfoil \\
& Mach .50 $\alpha 3^{\circ}$ & Mach $.50 \alpha 3^{\circ}$ & Mach .75 $\alpha 0^{\circ}$ \\
\hline $40 \times 8$ & .0099 & .0089 & .0126 \\
$80 \times 16$ & .0024 & .0017 & .0026 \\
$160 \times 32$ & .0002 & .0002 & .0001 \\
\hline
\end{tabular}

Table 5: Drag Coefficient on a sequence of meshes: H-characteristic scheme 


\begin{tabular}{|c|c|c|c|}
\hline Mesh & $\begin{array}{r}\text { RAE 2822 } \\
\text { Mach .50 } \alpha 3^{\circ}\end{array}$ & $\begin{array}{r}\text { NACA } 0012 \\
\text { Mach } .50 \propto 3^{\circ}\end{array}$ & $\begin{array}{r}\text { Korn Airfoil } \\
\text { Mach .75 } \alpha 0^{\circ}\end{array}$ \\
\hline $40 \times 8$ & .0062 & .0047 & .0098 \\
\hline $80 \times 16$ & .0013 & .0008 & .0017 \\
\hline $160 \times 32$ & .0000 & .0000 & .0000 \\
\hline
\end{tabular}

Table 6: Drag Coefficient on a sequence of meshes: H-CUSP scheme

compressible flow calculations. In the case of scalar conservation laws the LED property can be secured by corresponding symmetric and upstream limited positive (SLIP and USLIP) schemes.

The different scalar constructions can be combined with alternate numerical fluxes to provide a matrix of schemes for the gas dynamic equations. The property of supporting stationary discrete shocks with a single interior point is shared by the characteristic and CUSP schemes. Each of these schemes can be modified to preserve constant stagnation enthalpy in steady flows, giving four variants, the $\mathrm{E}$ and $\mathrm{H}$-characteristic schemes, and the $\mathrm{E}$ and H-CUSP schemes. The CUSP schemes are inexpensive. They introduce a minimum amount of numerical diffusion as the Mach number approaches zero. They are therefore also appropriate for viscous flow calculations in which it is important not to contaminate the boundary layer.

The theoretical properties of these schemes are verified by numerical calculations of one-dimensional, twodimensional and three-dimensional flows. References [11] and [12] evaluate the accuracy and efficiency of some of these schemes for the calculation of viscous flows.

\section{Acknowledgment}

This work has benefited from the generous support of ARPA under Grant No. N00014-92-J-1796, AFOSR under Grant No. AFOSR-91-0391, and IBM. The warm hospitality of the Aeronautics and Astronautics Department of Stanford University, and NASA Ames Research Center, provided a very favorable environment for the pursuit of this research while the author was on leave from Princeton University.

\section{References}

[1] H. Aiso. Admissibility of difference approximations for scalar conservation laws. Hiroshima Math. Journal, $23: 15-61,1993$.

[2] N. Balakrishnan and S. M. Deshpande. New upwind schemes with wave-particle splitting for inviscid compressible flows. Report 91 FM 12, Indian Institute of Science, 1991.

[3] B. Einfeldt. On Godunov-type methods for gas dynamics. SIAM J. Num. Anal., 25:294-318, 1988.

[4] A. Harten, P.D. Lax, and B. Van Leer. On upstream differencing and Godunov-type schemes for hyperbolic conservation laws. SIAM Review, 25:35-61, 1983.

[5] A. Jameson. Analysis and design of numerical schemes for gas dynamics 1, artificial diffusion, upwind biasing, limiters and their effect on multigrid convergence. Int. J. of Comp. Fluid Dyn., To Appear.

[6] B. Van Leer. Towards the ultimate conservative difference scheme. 11. Monotonicity and conservation combined in a second order scheme. J. Comp. Phys., 14:361-370, 1974.

[7] M-S. Liou and C.J. Steffen. A new flux splitting scheme. J. Comp. Phys., 107:23-39, 1993.

[8] S. V. Rao and S. M. Deshpande. A class of efficient kinetic upwind methods for compressible flows. Report 91 FM 11, Indian Institute of Science, 1991. 
[9] P.L. Roe. Approximate Riemann solvers, parameter vectors, and difference schemes. J. Comp. Phys., 43:357-372, 1981 .

[10] P.L. Roe. Fluctuations and signals - a framework for numerical evolution prolems. In K.W. Morton and M.J. Baines, editors, Proceedings of IMA Conference on Numerical Methods in Fluid Dynamics, pages 219-257, Reading, 1981.

[11] S. Tatsumi, L. Martinelli, and A. Jameson. Design, implementation, and validation of flux limited schemes for the solution of the compressible Navier-Stokes equations. AIAA paper 94-0647, AlAA 32nd Aerospace Sciences Meeting, Reno, Nevada, January 1994.

[12] S. Tatsumi, L. Martinelli, and A. Jameson. A new high resolution scheme for compressible viscous flows with shocks. AIAA paper To Appear, AIAA 33nd Aerospace Sciences Meeting, Reno, Nevada, January 1995.

[13] Y. Wada and M-S. Liou. A flux splitting scheme with high-resolution and robustness for discontinuities. AIAA paper 94-0083, AIAA 32nd Aerospace Sciences Meeting, Reno, Nevada, January 1994.

\section{Appendix: Eigenvalues and Eigenvectors for Gas Dynamic Equa- tions}

The Euler equations which describe the three-dimensional flow of an inviscid gas can be written as

$$
\frac{d}{d t} \int_{\Omega} W d V+\int_{\partial \Omega}\left(F_{x} d S_{x}+F_{y} d S_{y}+F_{z} d S_{z}\right)=0
$$

where $W$ is the state vector, $F_{x}, F_{y}$ and $F_{z}$ are the flux vectors, and $d S_{x}, d S_{y}$ and $d S_{z}$ are the projections of the surface element in the $x, y$ and $z$ coordinate directions. Let $u, v$ and $w$ be the velocity components and $\rho$, $p, E$ and $H$ the density, pressure, total energy and total enthalpy. Then

$$
W=\left\{\begin{array}{c}
\rho \\
\rho u \\
\rho v \\
\rho w \\
\rho E
\end{array}\right\}, F_{x}=\left\{\begin{array}{c}
\rho u \\
\rho u u+p \\
\rho u v \\
\rho u w \\
\rho u H
\end{array}\right\}, F_{y}=\left\{\begin{array}{c}
\rho v \\
\rho v u \\
\rho v v+p \\
\rho v w \\
\rho v H
\end{array}\right\}, F_{z}=\left\{\begin{array}{c}
\rho w \\
\rho w u \\
\rho w v \\
\rho w w+p \\
\rho w H
\end{array}\right\}
$$

Also,

$$
p=(\gamma-1) \rho\left(E-\frac{q^{2}}{2}\right), \quad H=E+\frac{p}{\rho}=\frac{c^{2}}{\gamma-1}+\frac{q^{2}}{2}
$$

where $q$ is the flow speed, and $c$ is the speed of sound,

$$
q^{2}=u^{2}+v^{2}+w^{2}, c^{2}=\frac{\gamma p}{\rho}
$$

When flow is smooth it can be represented by the quasi-linear differential equation

$$
\frac{\partial W}{\partial t}+A_{x} \frac{\partial W}{\partial x}+A_{y} \frac{\partial W}{\partial y}+A_{z} \frac{\partial W}{\partial z}=0
$$

where $A_{x}, A_{y}$ and $A_{z}$ are the Jacobian matrices

$$
A_{x}=\frac{\partial F_{x}}{\partial W}, A_{y}=\frac{\partial F_{y}}{\partial W}, A_{z}=\frac{\partial F_{z}}{\partial W}
$$

Under a change of variables to a new state vector $\tilde{W}$, equation (28) is transformed to

$$
\frac{\partial \tilde{W}}{\partial t}+\tilde{A}_{x} \frac{\partial \tilde{W}}{\partial x}+\tilde{A}_{y} \frac{\partial \tilde{W}}{\partial y}+\tilde{A}_{z} \frac{\partial \tilde{W}}{\partial z}=0
$$


where

$$
A_{x}=\tilde{M} \tilde{A}_{x} \tilde{M}^{-1}, \quad A_{y}=\tilde{M} \tilde{A}_{y} \tilde{M}^{-1}, \quad A_{z}=\tilde{M} \tilde{A}_{z} \tilde{M}^{-1}
$$

and

$$
\tilde{M}=\frac{\partial W}{\partial \tilde{W}}
$$

The finite volume discretization requires the evaluation of the flux through a face with vector area $S$,

$$
F=F_{x} S_{x}+F_{y} S_{y}+F_{z} S_{z} .
$$

The corresponding Jacobian matrix is

$$
A=\frac{\partial F}{\partial W}=S_{x} A_{x}+S_{y} A_{y}+S_{z} A_{z} .
$$

Let $\mathrm{S}$ be the magnitude of the face area

$$
S^{2}=S_{x}^{2}+S_{y}^{2}+S_{z}^{2}
$$

and $n_{x}, n_{y}$ and $n_{z}$ be the components of the unit normal

$$
n_{x}=\frac{S_{x}}{S}, n_{y}=\frac{S_{y}}{S}, n_{z}=\frac{S_{z}}{S}
$$

Also let $Q$ be the flux rate

$$
Q=u S_{x}+v S_{y}+w S_{z} .
$$

Then the Jacobian matrix can be decomposed as

$$
A=M \hat{A} M^{-1}
$$

where

$$
\hat{M}=\left(\begin{array}{ccccc}
1 & 0 & 0 & 0 & 0 \\
u & 1 & 0 & 0 & 0 \\
v & 0 & 1 & 0 & 0 \\
w & 0 & 0 & 1 & 0 \\
\frac{q^{2}}{2} & u & v & w & \frac{1}{\gamma-1}
\end{array}\right), \hat{M}^{-1}=\left(\begin{array}{ccccc}
1 & 0 & 0 & 0 & 0 \\
-u & 1 & 0 & 0 & 0 \\
-v & 0 & 1 & 0 & 0 \\
-w & 0 & 0 & 1 & 0 \\
(\gamma-1) \frac{q^{2}}{2} & -(\gamma-1) u & -(\gamma-1) v & -(\gamma-1) w & \gamma-1
\end{array}\right)
$$

and

$$
\hat{A}=\left(\begin{array}{ccccc}
Q & S_{x} & S_{y} & S_{z} & 0 \\
0 & Q & 0 & 0 & S_{x} \\
0 & 0 & Q & 0 & S_{y} \\
0 & 0 & 0 & Q & S_{z} \\
0 & S_{x} c^{2} & S_{y} c^{2} & S_{z} c^{2} & Q
\end{array}\right)
$$

Also the further transformation

$$
\hat{A}=D P \bar{A} P^{-1} D^{-1}
$$

where

$$
\begin{aligned}
D & =\left(\begin{array}{ccccc}
1 & 0 & 0 & 0 & 0 \\
0 & c & 0 & 0 & 0 \\
0 & 0 & c & 0 & 0 \\
0 & 0 & 0 & c & 0 \\
0 & 0 & 0 & 0 & c^{2}
\end{array}\right), D^{-1}=\left(\begin{array}{ccccc}
1 & 0 & 0 & 0 & 0 \\
0 & \frac{1}{c} & 0 & 0 & 0 \\
0 & 0 & \frac{1}{c} & 0 & 0 \\
0 & 0 & 0 & \frac{1}{c} & 0 \\
0 & 0 & 0 & 0 & \frac{1}{c^{2}}
\end{array}\right) \\
P & =\left(\begin{array}{lllll}
1 & 0 & 0 & 0 & 1 \\
0 & 1 & 0 & 0 & 0 \\
0 & 0 & 1 & 0 & 0 \\
0 & 0 & 0 & 1 & 0 \\
1 & 0 & 0 & 0 & 0
\end{array}\right), P^{-1}=\left(\begin{array}{ccccc}
0 & 0 & 0 & 0 & 1 \\
0 & 1 & 0 & 0 & 0 \\
0 & 0 & 1 & 0 & 0 \\
0 & 0 & 0 & 1 & 0 \\
1 & 0 & 0 & 0 & -1
\end{array}\right)
\end{aligned}
$$


produces the symmetric form

$$
\bar{A}=\left(\begin{array}{ccccc}
Q & S_{x} c & S_{y} c & S_{z} c & 0 \\
S_{x} c & Q & 0 & 0 & 0 \\
S_{y} c & 0 & Q & 0 & 0 \\
S_{z} c & 0 & 0 & Q & 0 \\
0 & 0 & 0 & 0 & Q
\end{array}\right)
$$

Then $\hat{A}, \bar{A}$, and $A$ can be decomposed as

$$
\hat{A}=\hat{R} \Lambda \hat{R}^{-1}, \bar{A}=\bar{R} \Lambda \bar{R}^{-1}, \quad A=R \Lambda R^{-1}
$$

where the diagonal matix $\Lambda$ contains the eigenvalues

$$
\Lambda=\left(\begin{array}{ccccc}
Q & 0 & 0 & 0 & 0 \\
0 & Q & 0 & 0 & 0 \\
0 & 0 & Q & 0 & 0 \\
0 & 0 & 0 & Q+c S & 0 \\
0 & 0 & 0 & 0 & Q-c S
\end{array}\right)
$$

Also the right eigenvectors of $\hat{A}, \bar{A}$, and $A$ are the columns of

$$
\hat{R}=D N, \quad \bar{R}=P^{-1} N, \quad R=\hat{M} D N
$$

and the left eigenvectors of $\hat{A}, \bar{A}$, and $A$ are the rows of

$$
\hat{R}^{-1}=N^{-1} D^{-1}, \quad \bar{R}^{-1}=N^{-1} P, \quad R^{-1}=N^{-1} D^{-1} \hat{M}^{-1}
$$

where

$$
N=\left(\begin{array}{ccccc}
n_{x} & n_{y} & n_{z} & 1 & 1 \\
0 & -n_{z} & n_{y} & n_{x} & -n_{x} \\
n_{z} & 0 & -n_{x} & n_{y} & -n_{y} \\
-n_{y} & n_{x} & 0 & n_{z} & -n_{z} \\
0 & 0 & 0 & 1 & 1
\end{array}\right), N^{-1}=\left(\begin{array}{ccccc}
n_{x} & 0 & n_{z} & -n_{y} & -n_{x} \\
n_{y} & -n_{z} & 0 & n_{x} & -n_{y} \\
n_{z} & n_{y} & -n_{x} & 0 & -n_{z} \\
0 & \frac{n_{x}}{2} & \frac{n_{y}}{2} & \frac{n_{x}}{2} & \frac{1}{2} \\
0 & -\frac{n_{x}}{2} & -\frac{n_{y}}{2} & -\frac{n_{x}}{2} & \frac{1}{2}
\end{array}\right)
$$

The decomposition to $\hat{A}$ corresponds to the introduction of primitive variables, scaled by a diagonal matrix,

$$
d \hat{W}=\hat{D} d \tilde{W}, \tilde{W}=\left(\begin{array}{c}
\rho \\
u \\
v \\
w \\
p
\end{array}\right), \hat{D}=\left(\begin{array}{ccccc}
1 & 0 & 0 & 0 & 0 \\
0 & \rho & 0 & 0 & 0 \\
0 & 0 & \rho & 0 & 0 \\
0 & 0 & 0 & \rho & 0 \\
0 & 0 & 0 & 0 & \rho
\end{array}\right)
$$

The decomposition to $\bar{A}$ corresponds to the introduction of symmetrizing variables $\mathrm{dW}$, defined in differential form, scaled by another diagonal matrix

$$
d \bar{W}=\bar{D} d \mathcal{W}, \mathcal{W}=\left(\begin{array}{c}
\frac{d \rho}{\rho c} \\
d u \\
d v \\
d w \\
d p-c^{2} d \rho
\end{array}\right), \bar{D}=\left(\begin{array}{ccccc}
\frac{\rho}{c} & 0 & 0 & 0 & 0 \\
0 & \rho & 0 & 0 & 0 \\
0 & 0 & \rho & 0 & 0 \\
0 & 0 & 0 & \frac{\rho}{c} & 0 \\
0 & 0 & 0 & 0 & -\frac{1}{c^{2}}
\end{array}\right)
$$

Multiplying out $\hat{M} D N$, the right eigenvectors of $A$ can be expressed as follows. Set

$$
v_{0}=\left(\begin{array}{c}
1 \\
u \\
v \\
w \\
\frac{q^{2}}{2}
\end{array}\right), v_{1}=\left(\begin{array}{c}
0 \\
0 \\
n_{z} \\
-n_{y} \\
v n_{z}-w n_{y}
\end{array}\right), v_{2}=\left(\begin{array}{c}
0 \\
-n_{y} \\
0 \\
n_{x} \\
w n_{x}-v n_{z}
\end{array}\right), v_{3}=\left(\begin{array}{c}
0 \\
n_{y} \\
-n_{x} \\
0 \\
u n_{y}-v n_{x}
\end{array}\right)
$$


Then the eigenvectors corresponding to the eigenvalue $Q$ are

$$
r_{1}=n_{x} v_{0}+v_{1}, r_{2}=n_{y} v_{0}+v_{2}, r_{3}=n_{z} v_{0}+v_{3}
$$

Here $v_{0}$ represents an entropy wave and $v_{12}$ represent vorticity waves. Also let $q_{n}$ denote the normal velocity $Q / S$, and set

$$
v_{4}=\left(\begin{array}{c}
1 \\
u \\
v \\
w \\
H
\end{array}\right), v_{5}=\left(\begin{array}{c}
0 \\
n_{x} \\
n_{y} \\
n_{z} \\
q_{n}
\end{array}\right)
$$

Then the last two eigenvectors, corresponding to pressure waves, are

$$
r_{4}=v_{4}+c v_{5}, r_{5}=v_{4}-c v_{5}
$$

The H-characteristic and H-CUSP schemes introduce the modified Jacobian matrix

$$
A_{h}=\frac{\partial F}{\partial w_{h}}
$$

where $w_{h}$ is the modified state vector

$$
w_{h}=\left(\begin{array}{c}
\rho \\
\rho u \\
\rho v \\
\rho w \\
\rho H
\end{array}\right)
$$

Using transformations of the same kind as those that were used for the standard Jacobian matrix, this can be decomposed as

$$
A_{h}=\hat{M}_{h} \hat{A}_{h} \hat{M}_{h}^{-1}
$$

where

$$
\hat{M}_{h}=\left(\begin{array}{ccccc}
1 & 0 & 0 & 0 & 0 \\
u & 1 & 0 & 0 & 0 \\
v & 0 & 1 & 0 & 0 \\
w & 0 & 0 & 1 & 0 \\
\frac{q^{2}}{2} & u & v & w & \frac{\gamma}{\gamma-1}
\end{array}\right), \hat{M}_{h}^{-1}=\left(\begin{array}{ccccc}
1 & 0 & 0 & 0 & 0 \\
-u & 1 & 0 & 0 & 0 \\
-v & 0 & 1 & 0 & 0 \\
-w & 0 & 0 & 1 & 0 \\
\frac{\gamma-1}{\gamma} \frac{q^{2}}{2} & -\frac{\gamma-1}{\gamma} u & -\frac{\gamma-1}{\gamma} v & -\frac{\gamma-1}{\gamma} w & \frac{\gamma-1}{\gamma}
\end{array}\right)
$$

and

$$
\hat{A}_{h}=\left(\begin{array}{ccccc}
Q & S_{x} & S_{y} & S_{z} & 0 \\
0 & Q & 0 & 0 & S_{x} \\
0 & 0 & Q & 0 & S_{y} \\
0 & 0 & 0 & Q & S_{z} \\
0 & S_{x} \frac{c^{2}}{\gamma} & S_{y} \frac{c^{2}}{\gamma} & S_{z} \frac{c^{2}}{\gamma} & \frac{Q}{\gamma}
\end{array}\right)
$$

Here

$$
\hat{M}_{h}=\hat{M} D_{h}, \quad \hat{M}_{h}^{-1}=D_{h}^{-1} \hat{M}^{-1}, \quad \hat{A}_{h}=D_{h}^{-1} \hat{A}
$$

where

$$
D_{h}=\left(\begin{array}{ccccc}
1 & 0 & 0 & 0 & 0 \\
0 & 1 & 0 & 0 & 0 \\
0 & 0 & 1 & 0 & 0 \\
0 & 0 & 0 & 1 & 0 \\
0 & 0 & 0 & 0 & \gamma
\end{array}\right)
$$


The eigenvalues of $\hat{A}_{h}$, and also those of $A_{h}$, since they can be derived from $\hat{A}_{h}$ by a similarity transformation, are $Q, Q, Q, \lambda^{+}$, and $\lambda^{-}$where

$$
\begin{aligned}
\lambda^{ \pm} & =\frac{\gamma+1}{2 \gamma} Q \pm \sqrt{\left(\frac{\gamma+1}{2 \gamma} Q\right)^{2}+\frac{c^{2} S^{2}-Q^{2}}{\gamma}} \\
& =\frac{\gamma+1}{2 \gamma} Q \pm \sqrt{\left(\frac{\gamma-1}{2 \gamma} Q\right)^{2}+\frac{c^{2} S^{2}}{\gamma}}
\end{aligned}
$$

Thus $\lambda^{+}$and $\lambda^{-}$change sign when $Q= \pm c S$, and $\lambda^{+}$has the same sign as $Q+c S$, while $\lambda^{-}$has the same sign as $Q-c S$. It is convenient to set

$$
\begin{aligned}
\sigma & =\frac{\gamma-1}{2 \gamma} \frac{Q}{c S}, \quad \mu=\sqrt{\sigma^{2}+\frac{1}{\gamma}} \\
\alpha^{+} & =\frac{\lambda^{+}-Q}{c S}=\mu-\sigma, \alpha^{-}=\frac{\lambda^{-}-Q}{c S}=-(\mu+\sigma)
\end{aligned}
$$

Now $\hat{A}_{h}$ can be decomposed as

$$
\hat{A}_{h}=\hat{R}_{h} \Lambda_{h} \hat{R}_{h}^{-1}
$$

where

$$
\Lambda_{h}=\left(\begin{array}{ccccc}
Q & 0 & 0 & 0 & 0 \\
0 & Q & 0 & 0 & 0 \\
0 & 0 & Q & 0 & 0 \\
0 & 0 & 0 & \lambda^{+} & 0 \\
0 & 0 & 0 & 0 & \lambda^{-}
\end{array}\right)
$$

Also

$$
\hat{R}_{h}=D N_{h}, \quad \hat{R}_{h}^{-1}=N_{h}^{-1} D^{-1}
$$

where $D$ is the same diagonal scaling matrix as before, and

$$
N_{h}=\left(\begin{array}{ccccc}
n_{x} & n_{y} & n_{z} & 1 & 1 \\
0 & -n_{z} & n_{y} & \alpha^{+} n_{x} & \alpha^{-} n_{x} \\
n_{z} & 0 & -n_{x} & \alpha^{+} n_{y} & \alpha^{-} n_{y} \\
-n_{y} & n_{x} & 0 & \alpha^{+} n_{z} & \alpha^{-} n_{z} \\
0 & 0 & 0 & \alpha^{+2} & \alpha^{-2}
\end{array}\right)
$$

with the inverse

$$
N_{h}^{-1}=\left(\begin{array}{ccccc}
n_{x} & -2 \gamma \sigma n_{x}^{2} & n_{z}-2 \gamma \sigma n_{y} n_{x} & -n_{y}-2 \gamma \sigma n_{z} n_{x} & \frac{n_{x}}{\alpha^{+} \alpha^{-}} \\
n_{y} & -n_{z}-2 \gamma \sigma n_{x} n_{y} & -2 \gamma \sigma n_{y}^{2} & n_{x}-2 \gamma \sigma n_{z} n_{y} & \frac{n_{y}}{\alpha^{+}} \\
n_{z} & n_{y}-2 \gamma \sigma n_{x} n_{z} & -n_{x}-2 \gamma \sigma n_{y} n_{z} & -2 \gamma \sigma n_{z}^{2} & \frac{n_{x}}{\alpha^{+} \alpha^{-}} \\
0 & \frac{-\alpha^{-}}{2 \mu \alpha^{+}} n_{x} & \frac{-\alpha^{-}}{2 \mu \alpha^{+}} n_{y} & \frac{-\alpha^{-}}{2 \mu \alpha^{+}} n_{z} & \frac{1}{2 \mu \alpha^{+}} \\
0 & \frac{\alpha^{+}}{2 \mu \alpha^{-}} n_{x} & \frac{\alpha^{+}}{2 \mu \alpha^{-}} n_{y} & \frac{\alpha^{+}}{2 \mu \alpha^{-}} n_{z} & \frac{-1}{2 \mu \alpha^{-}}
\end{array}\right)
$$

The formulas for the standard Jacobian matrix are recovered by setting $\alpha^{+}=1, \alpha^{-}=-1, \mu=\frac{1}{2}\left(\alpha^{+}-\alpha^{-}\right)=1$, $\sigma=-\frac{1}{2}\left(\alpha^{+}+\alpha^{-}\right)=0$. Correspondingly, $A_{h}$ can now be represented as

$$
A_{h}=R_{h} \Lambda_{h} R_{h}^{-1}
$$

where the right eigenvectors of $A_{h}$ are the columns of

$$
R_{h}=\hat{M}_{h} \hat{R}_{h}=\hat{M}_{h} D N_{h}
$$

and the left eigenvectors of $A_{h}$ are the rows of

$$
R_{h}^{-1}=\hat{R}_{h}^{-1} \hat{M}_{h}^{-1}=N_{h}^{-1} D^{-1} \hat{M}_{h}^{-1}
$$

These decompositions of $A$ and $A_{h}$ express every element in terms of velocities and metric quantities, while the density is completely eliminated from the formulas. 


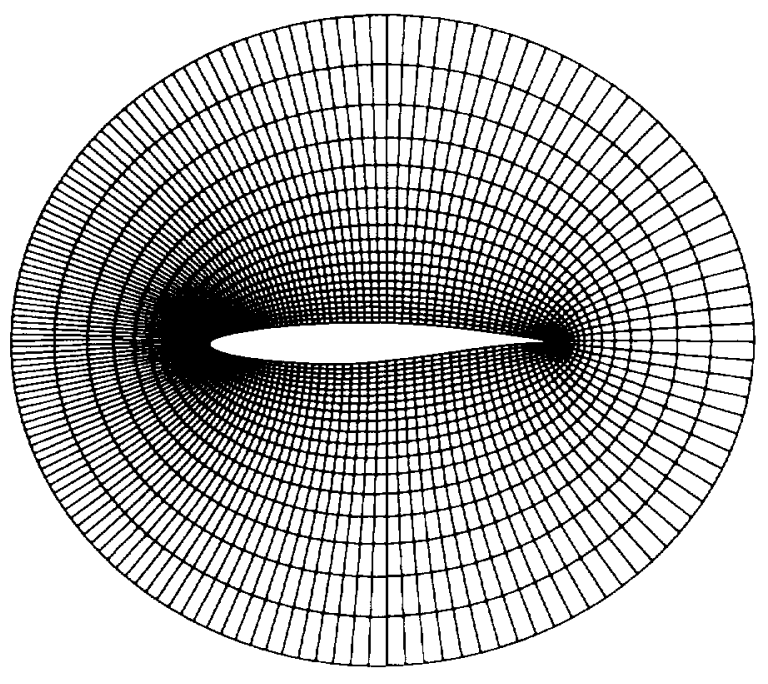

2a: RAE-2822 Airfoil

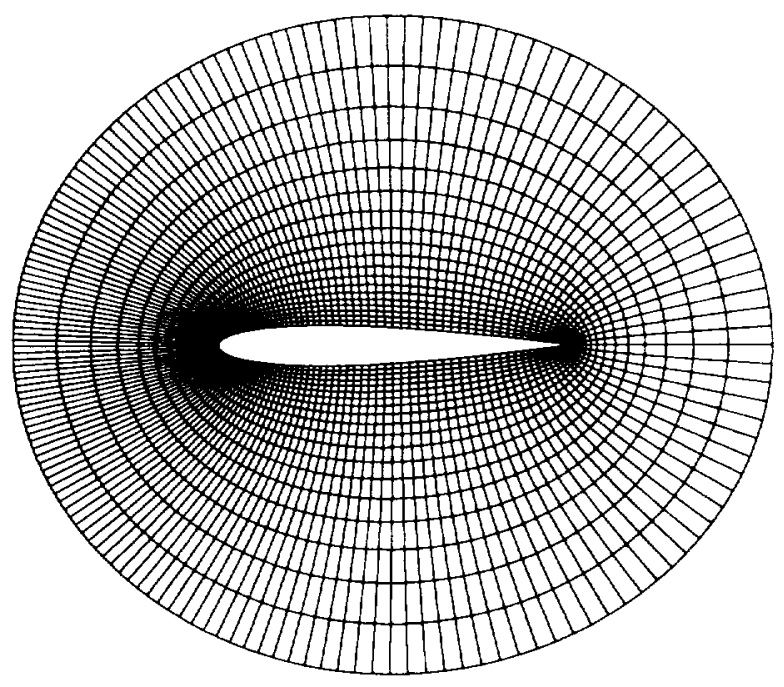

2b: NACA-0012 Airfoil

Figure 2: O-Topology Meshes, 160x32 


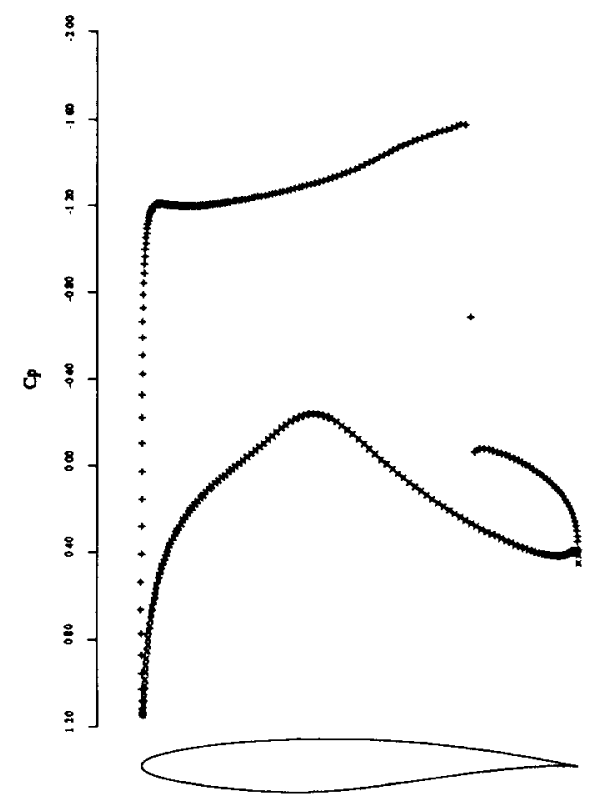

3a: $C_{p}$ after 25 Cycles.

$C_{l}=1.1227, C_{d}=0.0460$.

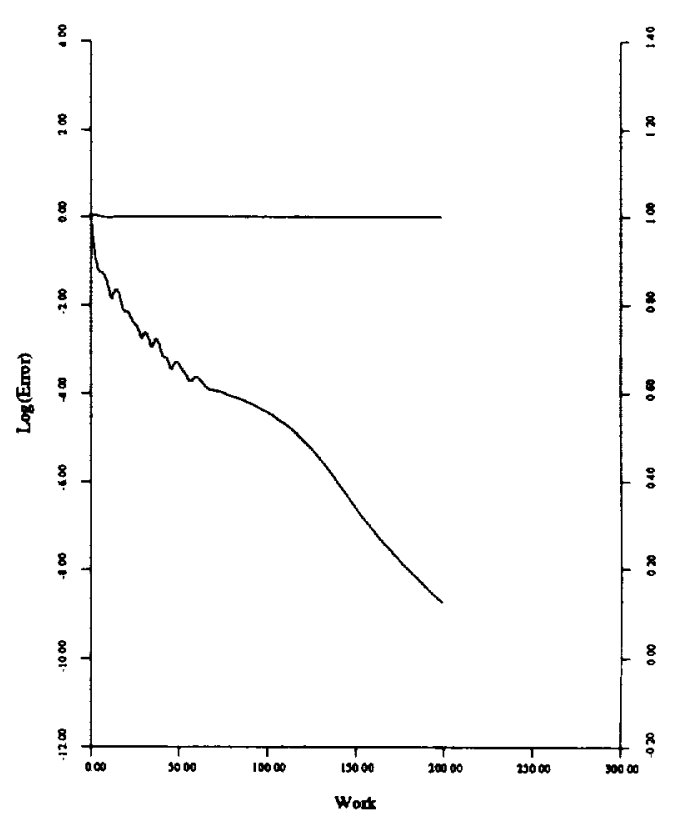

3b: Convergence.

Figure 3: RAE-2822 Airfoil at Mach 0.750 and $\alpha=3.0^{\circ}$

H-characteristic Scheme.

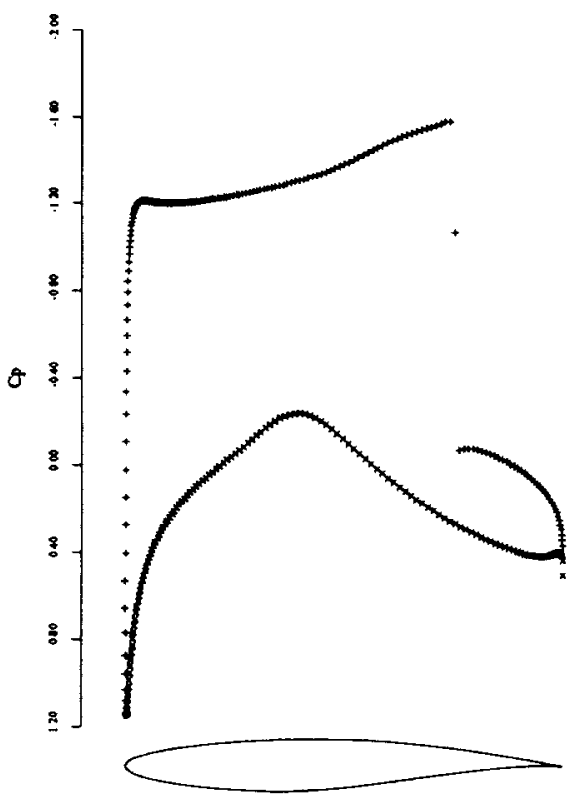

4a: $C_{p}$ after 25 Cycles. $C_{l}=1.1312, C_{d}=0.0469$.

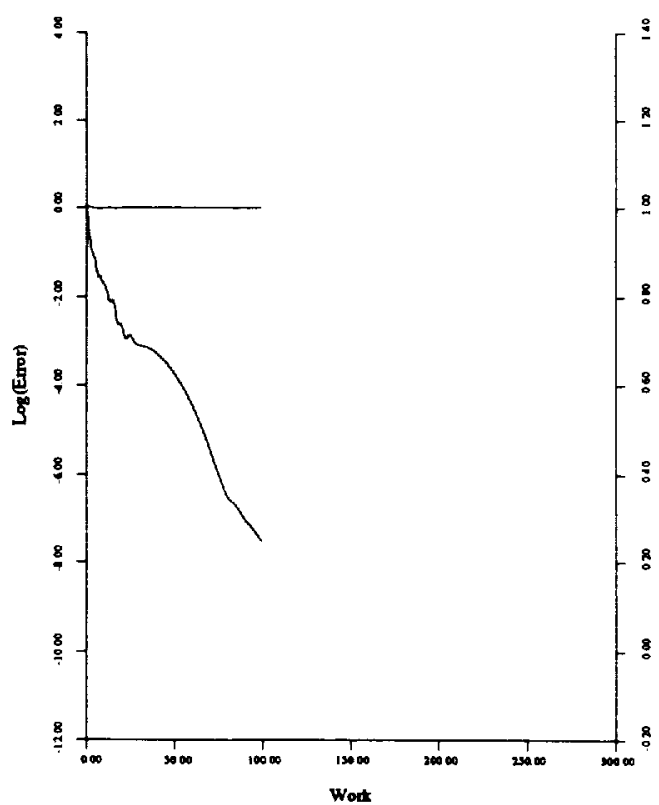

4b: Convergence.

Figure 4: RAE-2822 Airfoil at Mach 0.750 and $\alpha=3.0^{\circ}$

H-CUSP Scheme. 


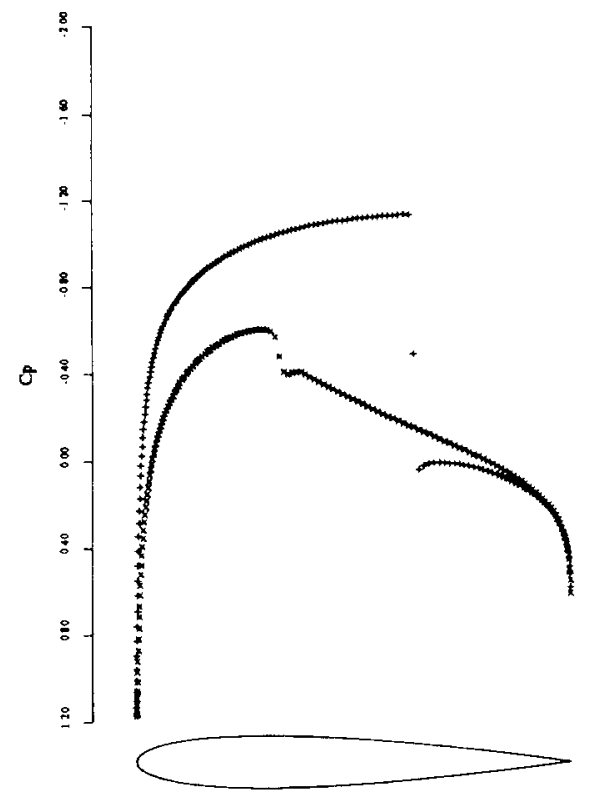

5a: $C_{p}$ after 75 Cycles.

$C_{l}=0.3620, C_{d}=0.0230$.

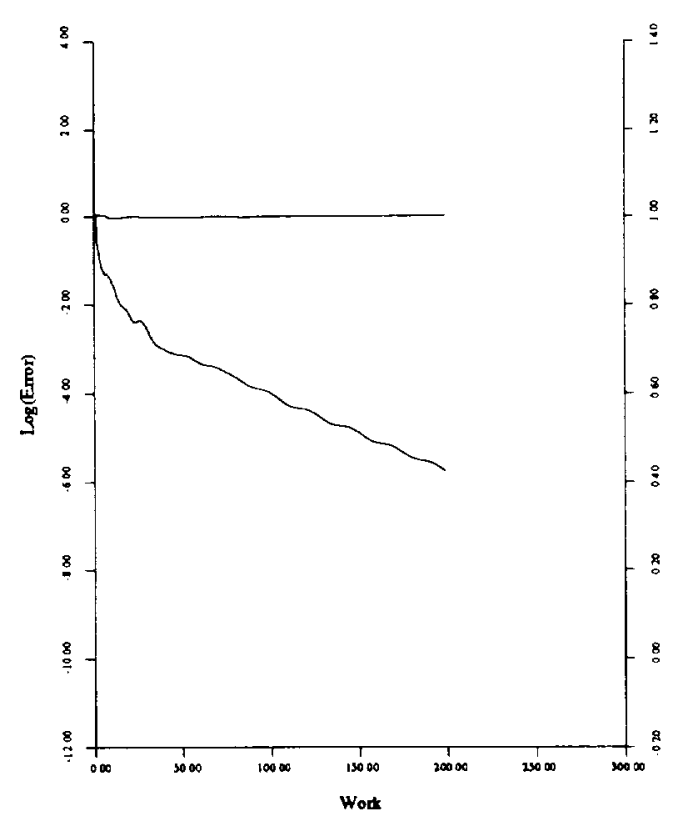

5b: Convergence.

Figure 5: NACA-0012 Airfoil at Mach 0.800 and $\alpha=1.25^{\circ}$

$\mathrm{H}$-characteristic Scheme.

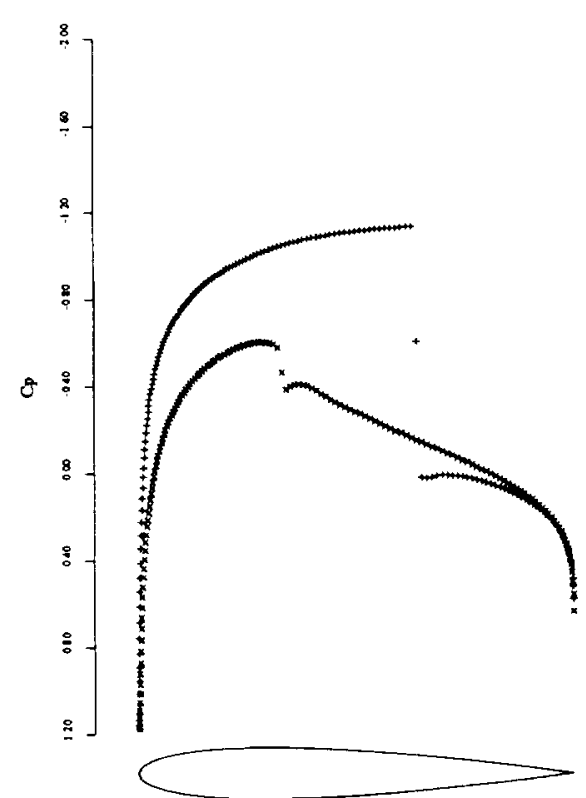

6a: $C_{p}$ after 35 Cycles. $C_{l}=0.3654, C_{d}=0.0232$.

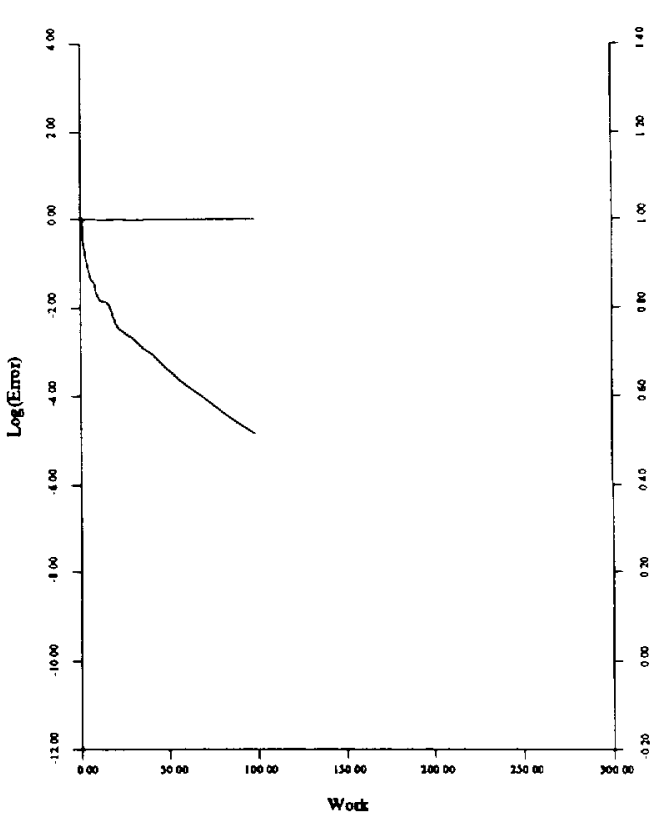

6b: Convergence.

Figure 6: NACA-0012 Airfoil at Mach 0.800 and $\alpha=1.25^{\circ}$

H-CUSP Scheme. 


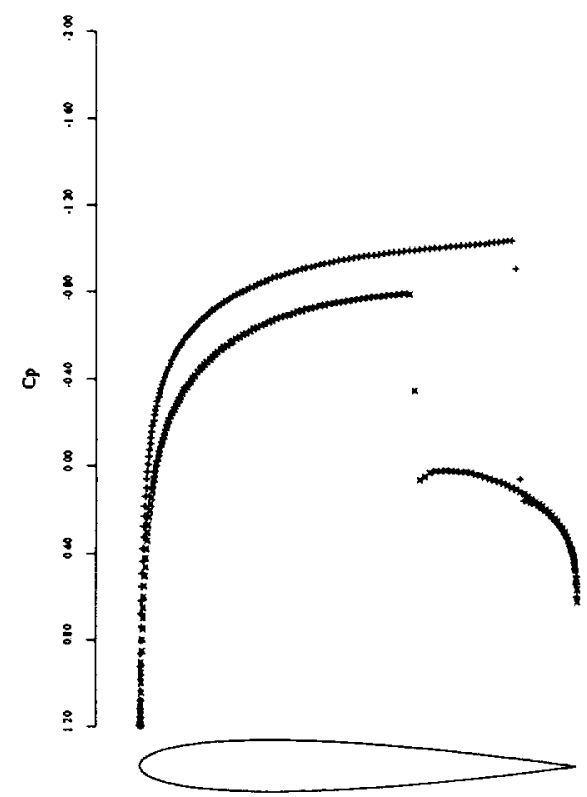

7a: $C_{p}$ after 75 Cycles.

$C_{l}=0.3818, C_{d}=0.0580$.

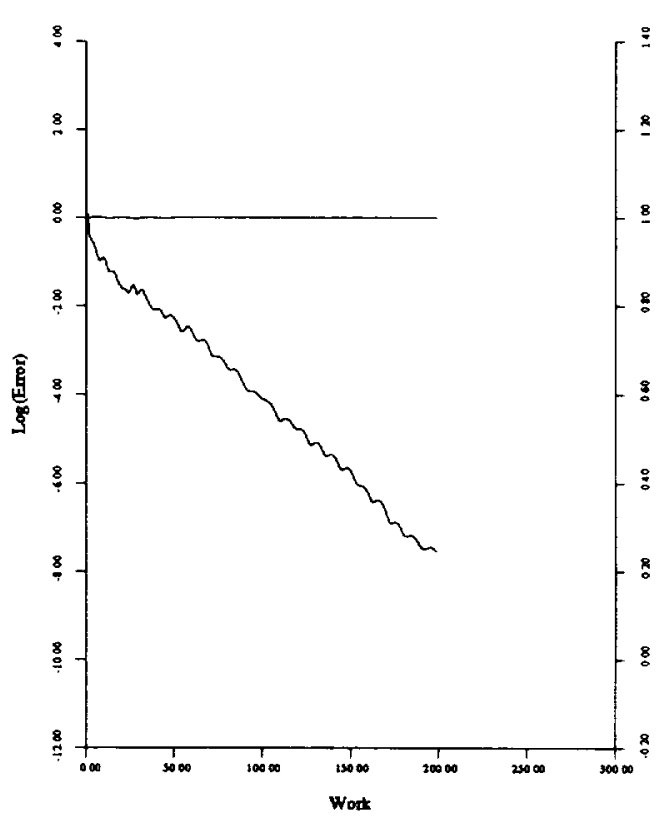

7b: Convergence.

Figure 7: NACA-0012 Airfoil at Mach 0.850 and $\alpha=1.0^{\circ}$ H-characteristic Scheme.

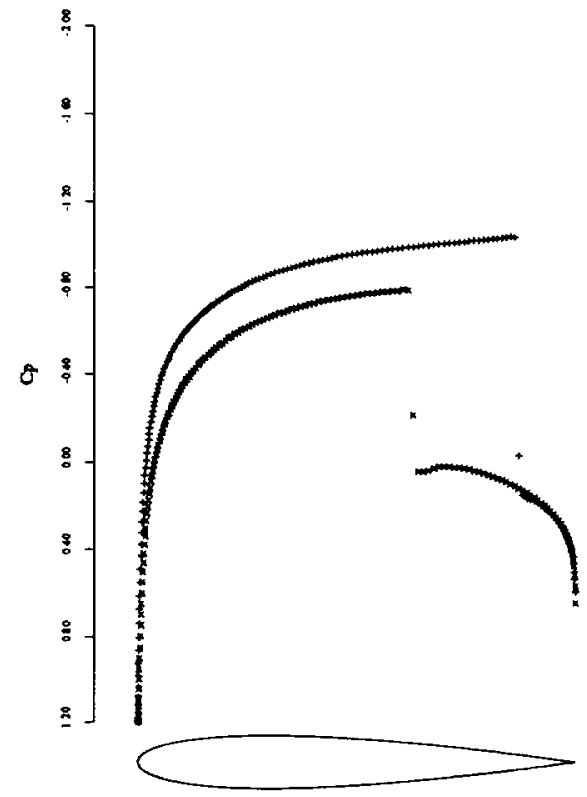

8a: $C_{p}$ after 35 Cycles. $C_{l}=0.3861, C_{d}=0.0582$.

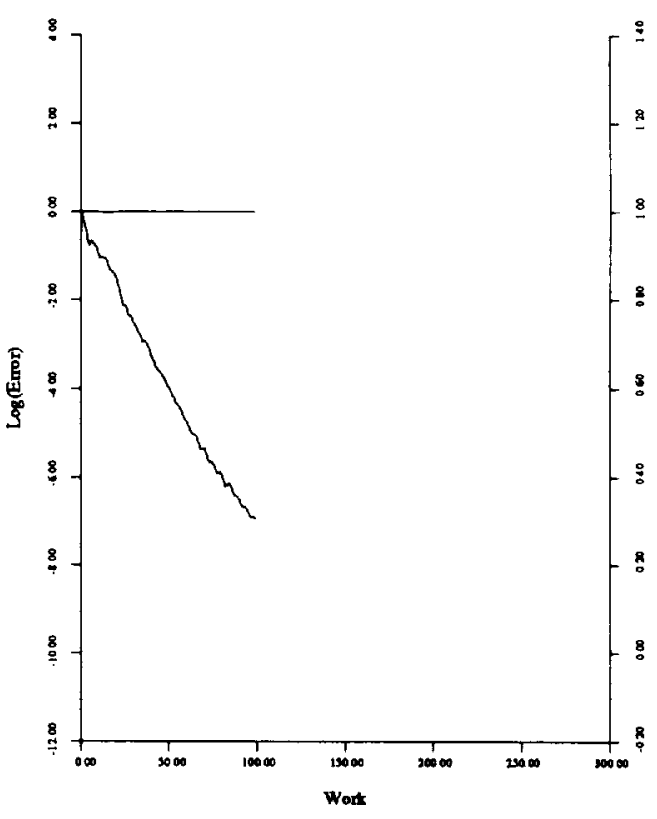

$8 \mathrm{~b}$ : Convergence.

Figure 8: NACA-0012 Airfoil at Mach 0.850 and $\alpha=1.0^{\circ}$

H-CUSP Scheme. 


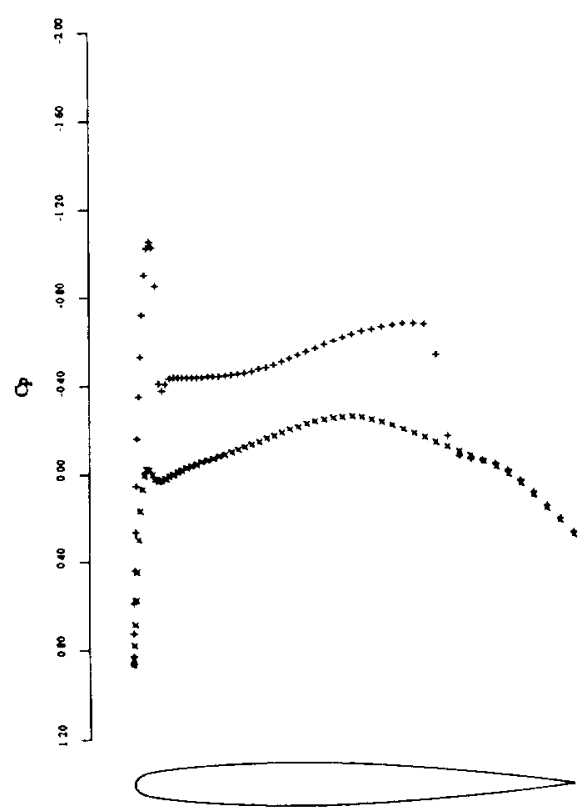

9a: $12.50 \%$ Span.

$C_{l}=0.2933, C_{d}=0.0274$.

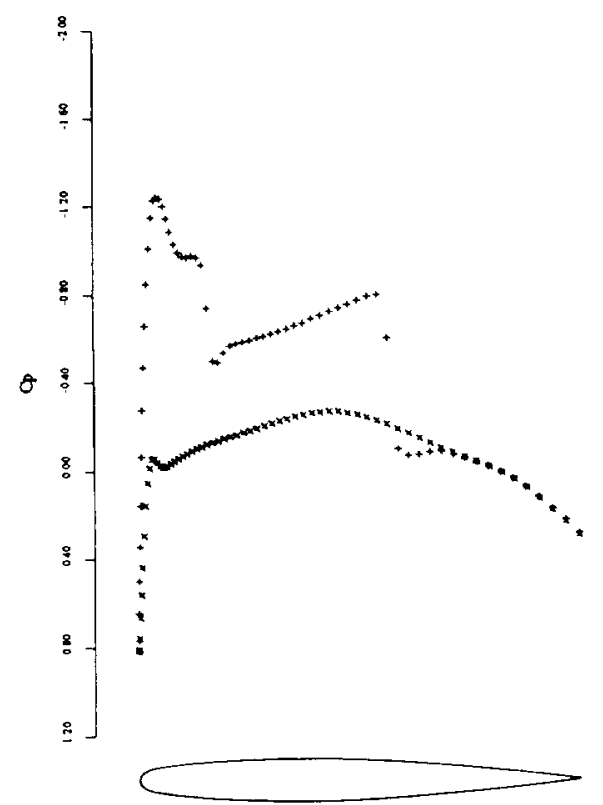

9c: $50.00 \%$ Span.

$C_{l}=0.3262, C_{d}=0.0089$.

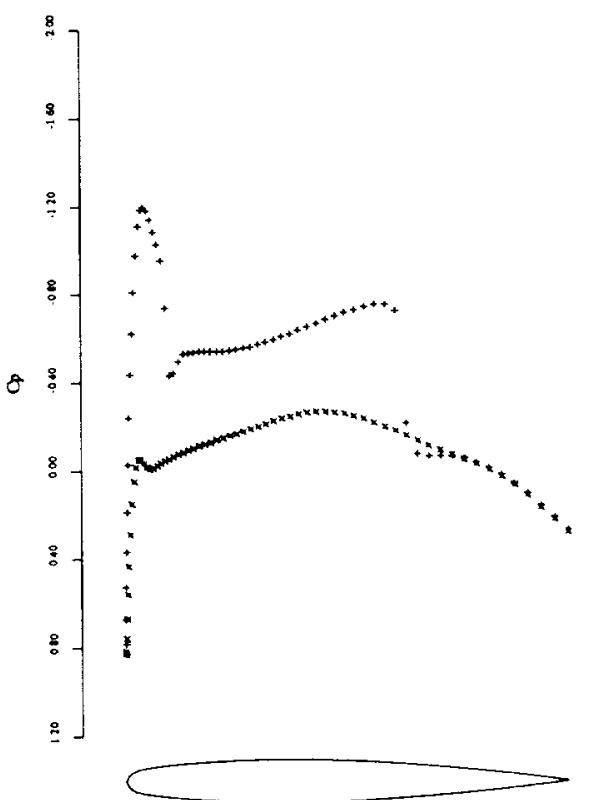

9b: $31.25 \%$ Span.

$C_{l}=0.3139, C_{d}=0.0159$.

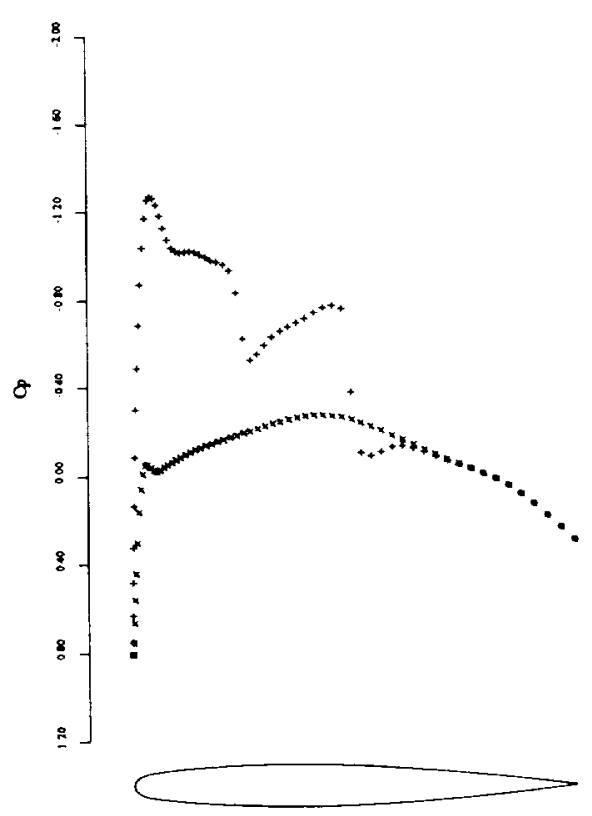

9d: $68.75 \%$ Span.

$C_{l}=0.3195, C_{d}=0.0026$.

Figure 9: Onera M6 Wing.

Mach 0.840 , Angle of Attack $3.06^{\circ}, 192 \times 32 \times 48$ Mesh.

$C_{L}=0.3041, C_{D}=0.0131$.

H-CUSP scheme. 
root ra-iris12.arc.nasa.gov Super-User reuther@ra-iris18.arc.nasa.gov: covermdo.ps

Wed Sep 14 19:07:04 PDT 1994

rajet rajet-8787 Hewlett-Packard LaserJet IIISi

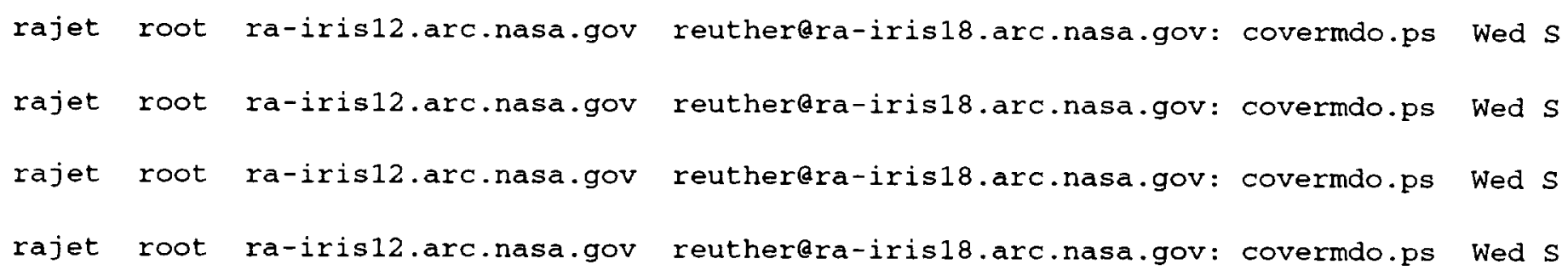

